TUM-HEP-608/05

KYUSHU-HET-79

hep-ph/0511108

\title{
Discrete Flavor Symmetry, Dynamical Mass Textures, and Grand Unification
}

\author{
Naoyuki Haba ${ }^{\mathrm{a}}$ and Koichi Yoshioka ${ }^{\mathrm{b}}$ \\ a Physik-Department, Technische Universität München, \\ James-Franck-Straße, 85748 Garching, Germany \\ ${ }^{\mathrm{b}}$ Department of Physics, Kyushu University, Fukuoka, 812-8581, Japan
}

(November, 2005)

\begin{abstract}
Discrete flavor symmetry is explored for an intrinsic property of mass matrix forms of quarks and leptons. In this paper we investigate the $S_{3}$ permutation symmetry and derive the general forms of mass matrices in various types of $S_{3}$ theories. We also exhibit particular realizations of previous ansatze of mass matrices, which have often been applied in the literature to the standard model Yukawa sector. Discrete flavor symmetry is also advantageous for vanishing matrix elements being dynamically generated in the vacuum of scalar potential. This is due to the fact that group operations are discrete. While zero elements themselves do not explain mass hierarchies, we introduce an abelian flavor symmetry. A non-trivial issue is whether successful quantum numbers can be assigned so that they are compatible with other (non-abelian) flavor symmetries. We show typical examples of charge assignments which not only produce hierarchical orders of mass eigenvalues but also prohibit non-renormalizable operators which disturb the hierarchies in first-order estimation. As an explicit application, a flavor model is constructed in grand unification scheme with $S_{3}$ and $U(1)$ (or $Z_{N}$ ) flavor symmetries.
\end{abstract}

\footnotetext{
${ }^{\mathrm{a}}$ On leave of absence from Institute of Theoretical Physics, University of Tokushima, 770-8502, Japan.
} 


\section{Introduction}

One of the most important issues in and beyond the standard model is the masses and mixing angles of the three-family quarks and leptons. After the electroweak symmetry breaking, the observed values of masses and mixing angles are to be produced from the structures of Yukawa couplings. However the Yukawa couplings generally have redundancy in explaining the experimental results: apparently different forms of Yukawa matrices lead to the same physical masses and mixing angles. Therefore it has been a long outstanding problem which patterns of Yukawa couplings are relevant from phenomenological and theoretical viewpoints. Various progresses have been made in the literature by applying additional principles to the standard-model Yukawa sector. The two well-known examples of such principles are to adopt the unification hypothesis of matter multiplets and to assume specific forms of Yukawa couplings ("textures"). The former is a top-down approach to the problem. The grand unification principle relates the properties of quarks and leptons, and reduces the degrees of freedom of Yukawa couplings in the theory. On the other hand, the latter approach is rather a bottom-up one. Available forms of Yukawa textures are explored so that they are consistent with the experimental observations. It is interesting that the number of successful textures is found to be highly limited. This fact is revealed from a simplifying assumption that some elements of Yukawa matrices vanish, called texture zeros. Along this line, phenomenologically possible forms of mass matrices have been proposed in the literature (for example, [1, 2, 3], and also [4, 5] for systematic analyses of zero textures).

As for the neutrino sector, the recent experimental results suggest that one of the most likely forms of Majorana mass textures of light neutrinos is proportional to

$$
\left(\begin{array}{ll}
\mathcal{O}(1) & \mathcal{O}(1) \\
\mathcal{O}(1) & \mathcal{O}(1)
\end{array}\right)
$$

in the basis where the generation mixing has been rotated out in the charged-lepton side. This is the dominant part of mass matrix and other small entries have not explicitly been written down. It is clearly seen that the large leptonic mixing between the second and third generations [6] is predicted from (1.1). The observed large 1-2 mixing [7] requires an additional condition for the above texture: the dominant $2 \times 2$ sub-matrix of (1.1) has a reduced rank and its determinant is of the same order of small off-diagonal elements neglected in (1.1). Given that, the texture form (1.1) is considered as a promising candidate consistent to the present experimental data. The condition (the vanishing determinant of the dominant sub-matrix) may be realized without fine tuning of model parameters, e.g., with the right-handed neutrino dominance [8], $R$-parity violation [9], 
and the lopsided form of charged-lepton mass matrix [10]. It is interesting that the lopsided mass textures can be naturally embedded in grand unified theory. However if the theory is supersymmetrized, large off-diagonal elements in the lepton Yukawa matrices generally induce sizable rates of flavor-violating processes to excess the present experimental bounds [1]. While the details depend on superparticle mass spectrum, a natural way to avoid this flavor problem is to consider the case that the lepton as well as quark Yukawa matrices take hierarchical forms, which lead to small generation mixing. If this is the case, an interesting possibility to have large lepton mixing is to suppose asymmetric forms of mass textures (zeros).* It seems that abelian flavor symmetry may be difficult to generate such asymmetric zeros without expense of model complexity. Furthermore non-abelian continuous flavor symmetry is not suitable for handling mass textures since texture zeros are rotated to other arbitrary forms by continuous symmetry rotations and do not have physical implications.

Motivated by these results, in this paper we investigate the power of non-abelian discrete flavor symmetry for constructing mass matrix models. We particularly focus on the minimal discrete non-abelian symmetry $S_{3}$. (For fermion mass models based on other non-minimal discrete non-abelian flavor symmetries, see [13].) The $S_{3}$ operation is the permutation of three objects, which has a simple geometrical interpretation, i.e. the symmetry of an equilateral triangle. While it is the smallest non-abelian discrete symmetry, it might be regarded as a remnant of flavor symmetry of fundamental theory in high-energy regime. The purposes of this paper are the following two points: (i) non-abelian flavor symmetries such as $S_{3}$ are so effective that various types of phenomenological textures are handled and (ii) these symmetries can also generate asymmetric forms of Yukawa matrices in dynamical ways. Further it should be noted that texture zeros themselves do not explain fermion mass hierarchies. Previous approaches to fermion masses with $S_{3}$ have assumed hierarchical values of Yukawa couplings and/or involved symmetry-breaking patterns such as vacuum expectation values (VEVs) of Higgs scalars. In this paper we show that realistic values of masses and mixing angles are dynamically achieved by introducing $U(1)$ symmetry. A non-trivial problem arises whether successful $U(1)$ quantum numbers can be assigned so that they are compatible with other non-abelian (flavor) symmetries. It is noticed that mass hierarchy is also realized in a similar way with a discrete subgroup of the flavor $U(1)$ symmetry such as $Z_{N}$ with appropriate (enough large) $N$ and the same quantum numbers as in the $U(1)$ case. A smaller choice of $N$ would be possible and interesting from a viewpoint of brevity. In this case, the problem of fermion masses can be handled with flavor symmetries that are entirely discrete. While the $U(1)$ charge as-

*Leptonic mixing angles may be enhanced, e.g. by integrating out heavy fields such as right-handed neutrinos [12]. 
signments are presented in this paper, they can always be read as the charges in flavor $Z_{N}$ theory. We finally present an explicit model where the $S_{3}$ flavor symmetry is incorporated consistently to unified gauge symmetry and hierarchical forms of mass matrices.

This paper is structured as follows. In the next section, we discuss some fundamental issues of the $S_{3}$ group, which are needed in Section 3 to study symmetry-invariant forms of mass matrices. In Section $4, S_{3}$ is applied to supply dynamical justifications to realistic candidates of mass textures which have often been discussed in the literature. Based on these results, we present in Section 5 a toy flavor model in grand unification scheme where Yukawa textures are controlled by a single flavor $S_{3}$, assisted by $U(1)$ symmetry. In Section 6, we analyze the invariant scalar potentials of $S_{3}$ doublet whose VEV form is a key ingredient of the approach developed in this paper. Section 7 is devoted to summarizing our results.

\section{The $S_{3}$ group}

\subsection{Representations and representation matrices}

The $S_{3}$ symmetry is the smallest non-abelian symmetry, the permutations which an equilateral triangle has. The $S_{3}$ group therefore contains six elements $T_{1}, \cdots, T_{6}$, half of which are the circulations of triangle apices and the other half corresponds to the exchanges of two of three apices while the other is fixed. There are only few numbers of irreducible representations; a two-dimensional representation and two different onedimensional representations. Throughout of this paper we denote them as 2 (doublet), $1_{\mathrm{S}}$ (singlet), and $1_{\mathrm{A}}$ (pseudo singlet), respectively. The non-trivial one-dimensional representation $1_{\mathrm{A}}$ distinguishes the group elements in two parts. The matrix representations of group elements are given in Table 1. It is easily seen that the even permutations $T_{1}$,

\begin{tabular}{c|c|c|c|c|c|c} 
& $T_{1}$ & $T_{2}$ & $T_{3}$ & $T_{4}$ & $T_{5}$ & $T_{6}$ \\
\hline 2 & $\left(\begin{array}{ll}1 & 0 \\
0 & 1\end{array}\right)$ & $\left(\begin{array}{cc}\chi & 0 \\
0 & \chi^{2}\end{array}\right)$ & $\left(\begin{array}{cc}\chi^{2} & 0 \\
0 & \chi\end{array}\right)$ & $\left(\begin{array}{ll}0 & 1 \\
1 & 0\end{array}\right)$ & $\left(\begin{array}{cc}0 & \chi^{2} \\
\chi & 0\end{array}\right)$ & $\left(\begin{array}{cc}0 & \chi \\
\chi^{2} & 0\end{array}\right)$ \\
\hline $1_{\mathrm{S}}$ & 1 & 1 & 1 & 1 & 1 & 1 \\
\hline $1_{\mathrm{A}}$ & 1 & 1 & 1 & -1 & -1 & -1
\end{tabular}

Table 1: The representation matrices of the $S_{3}$ elements. The symbol $\chi$ is the third root of unity $\left(\chi=e^{2 \pi i / 3}\right)$.

$T_{2}$ and $T_{3}$ constitute the subgroup $Z_{3}$. This means that the $S_{3}$ symmetry is broken down to $Z_{3}$ when a pseudo-singlet field $1_{\mathrm{A}}$ develops an expectation value. Thus the $S_{3}$ group 
has simple but non-trivial structures, and is suitable for applying it to the flavor problems of three-generation fermions in the standard model. A number of models have been proposed to explain Yukawa coupling structures of quarks and leptons with the $S_{3}$ flavor symmetry [14, 15, 16].

It may be convenient to introduce the reducible three-dimensional representations for discussing the three-generation flavor physics. Corresponding to $1_{\mathrm{S}}$ and $1_{\mathrm{A}}$, there are two types of three-dimensional representations:

$$
\begin{array}{ll}
3_{\mathrm{S}} \equiv 2+1_{\mathrm{S}}, & \text { (triplet) } \\
3_{\mathrm{A}} \equiv 2+1_{\mathrm{A}} . & \text { (pseudo triplet) }
\end{array}
$$

If $S_{3}$ is regarded as a remnant of some gauge symmetry in fundamental theory, $3_{\mathrm{A}}$ should be applied not to induce discrete gauge anomaly. This is understood from the fact that the three-dimensional vector representation of $S O(3)$ is decomposed as $3=2+1_{\mathrm{A}}$ in terms of its subgroup $S_{3}$. One may also use the $3_{\mathrm{S}}$ representation at the expense that $S_{3}$ is assumed to be a global symmetry or the anomaly is cancelled by introducing appropriate numbers of pseudo singlet fermions. Further the aforementioned geometrical interpretation of $S_{3}$ operations is made clear for triplet representations. Such an interpretation is seen in a different basis of $S_{3}$, as will be discussed later in this section. The matrix representations of $3_{\mathrm{S}}$ and $3_{\mathrm{A}}$ are read from Table 1 and given by

$$
\begin{aligned}
& T_{1}=\left(\begin{array}{ccc}
1 & & \\
& 1 & \\
& & 1
\end{array}\right), \quad T_{2}=\left(\begin{array}{lll}
\chi & & \\
& \chi^{2} & \\
& & 1
\end{array}\right), \quad T_{3}=\left(\begin{array}{lll}
\chi^{2} & & \\
& \chi & \\
& & 1
\end{array}\right), \\
& T_{4}=\left(\begin{array}{lll} 
& 1 & \\
1 & & \\
& & 1
\end{array}\right), \quad T_{5}=\left(\begin{array}{lll} 
& \chi^{2} & \\
\chi & & \\
& & 1
\end{array}\right), \quad T_{6}=\left(\begin{array}{lll} 
& \chi & \\
\chi^{2} & & \\
& & 1
\end{array}\right)
\end{aligned}
$$

for the $3_{\mathrm{S}}$ representation. As for $3_{\mathrm{A}}$, the matrices $T_{i}$ are given by (2.3) except that the 3 -3 elements in the odd permutation matrices $T_{4,5,6}$ are replaced with -1 .

\subsection{Tensor products and $2^{*}$ representation}

The tensor products involving $1_{\mathrm{A}}$ are given by $1_{\mathrm{A}} \times 1_{\mathrm{A}}=1_{\mathrm{S}}$ and $1_{\mathrm{A}} \times 2=2$. The only remaining non-trivial product is that of two doublets: $2 \times 2=2+1_{\mathrm{A}}+1_{\mathrm{S}}$. In the basis where the group elements are given by Table 1, the product of two doublets $\psi=\left(\psi_{1}, \psi_{2}\right)^{\mathrm{t}}$ and $\phi=\left(\phi_{1}, \phi_{2}\right)^{\mathrm{t}}$ is explicitly written as follows:

$$
\begin{aligned}
\psi \times \phi & =\left(\psi^{\dagger} \sigma_{+} \phi, \psi^{\dagger} \sigma_{-} \phi\right)_{2}^{\mathrm{t}}+\left(\psi^{\dagger} \sigma_{3} \phi\right)_{1_{\mathrm{A}}}+\left(\psi^{\dagger} \phi\right)_{1_{\mathrm{S}}}, \\
& =\left(\psi_{1}^{\dagger} \phi_{2}, \psi_{2}^{\dagger} \phi_{1}\right)_{2}^{\mathrm{t}}+\left(\psi_{1}^{\dagger} \phi_{1}-\psi_{2}^{\dagger} \phi_{2}\right)_{1_{\mathrm{A}}}+\left(\psi_{1}^{\dagger} \phi_{1}+\psi_{2}^{\dagger} \phi_{2}\right)_{1_{\mathrm{S}}}
\end{aligned}
$$


where $\sigma_{i}(i=1,2,3)$ are the Pauli matrices and $\sigma_{ \pm} \equiv \frac{\sigma_{1} \pm i \sigma_{2}}{2}$. The subscripts in the right-handed sides denote $S_{3}$ representations. Note that $\psi$ (and also $\phi$ ) is generically complex-valued, while $S_{3}$ is a real group. This fact is important when $\psi$ transforms as some complex representation under other symmetries than $S_{3}$. The complex conjugate $\psi^{*}$ belongs to the $2^{*}$ representation for which the representation matrices become $T_{i}^{*}$. In practical use, however, it is convenient to define a doublet from an anti-doublet:

$$
\psi_{C} \equiv \sigma_{1} \psi^{*}=\left(\begin{array}{c}
\psi_{2}^{*} \\
\psi_{1}^{*}
\end{array}\right),
$$

which transforms as $\psi_{C} \rightarrow \sigma_{1}\left(T_{i} \psi\right)^{*}=T_{i} \sigma_{1} \psi^{*}=T_{i} \psi_{C}$, and indeed acts as the 2 representation of $S_{3}$. The tensor product involving $\psi_{C}$ is hence given by

$$
\begin{aligned}
\psi_{C} \times \phi & =\left(\psi^{\mathrm{t}} \sigma_{L} \phi, \psi^{\mathrm{t}} \sigma_{R} \phi\right)_{2}^{\mathrm{t}}+\left(\psi^{\mathrm{t}} i \sigma_{2} \phi\right)_{1_{\mathrm{A}}}+\left(\psi^{\mathrm{t}} \sigma_{1} \phi\right)_{1_{\mathrm{S}}} \\
& =\left(\psi_{2} \phi_{2}, \psi_{1} \phi_{1}\right)_{2}^{\mathrm{t}}+\left(\psi_{1} \phi_{2}-\psi_{2} \phi_{1}\right)_{1_{\mathrm{A}}}+\left(\psi_{1} \phi_{2}+\psi_{2} \phi_{1}\right)_{1_{\mathrm{S}}}
\end{aligned}
$$

where $\sigma_{L} \equiv \frac{1-\sigma_{3}}{2}$ and $\sigma_{R} \equiv \frac{1+\sigma_{3}}{2}$, respectively. It is noticed that this product does not contain any complex conjugates. Such a type of tensor product is necessary for describing, e.g., matter interaction terms ( $F$ terms) in supersymmetric theory and Majorana masses for neutrinos. On the other hand, the product (2.4) is applied to the usual Dirac mass terms of quarks and leptons. The form of tensor product depends on the group basis, and therefore the $S_{3}$ model construction and its physical consequences also do. For details, see the next subsection and Section 3 .

It may be useful for later discussion to explicitly write down what types of singlet components are contained in the products of more than two doublets. In the basis discussed here, one finds

$$
\begin{array}{llll}
\psi \times \phi & & \psi_{1}^{\dagger} \phi_{1}+\psi_{2}^{\dagger} \phi_{2}, & \psi_{1} \phi_{2}+\psi_{2} \phi_{1}, \\
\psi \times \phi \times \varphi & \supset & \psi_{1}^{\dagger} \phi_{2} \varphi_{2}+\psi_{2}^{\dagger} \phi_{1} \varphi_{1}, \quad \psi_{1} \phi_{2}^{\dagger} \varphi_{1}+\psi_{2} \phi_{1}^{\dagger} \varphi_{2}, \\
& \psi_{1} \phi_{1} \varphi_{2}^{\dagger}+\psi_{2} \phi_{2} \varphi_{1}^{\dagger}, \quad \psi_{1} \phi_{1} \varphi_{1}+\psi_{2} \phi_{2} \varphi_{2}, \\
\psi \times \phi \times \varphi \times \chi & \supset & \psi_{1}^{\dagger} \phi_{1}^{\dagger} \varphi_{1} \chi_{1}+\psi_{2}^{\dagger} \phi_{2}^{\dagger} \varphi_{2} \chi_{2}, & \psi_{1} \phi_{1}^{\dagger} \varphi_{1}^{\dagger} \chi_{1}+\psi_{2} \phi_{2}^{\dagger} \varphi_{2}^{\dagger} \chi_{2}, \\
& \psi_{1} \phi_{1}^{\dagger} \varphi_{1} \chi_{1}^{\dagger}+\psi_{2} \phi_{2}^{\dagger} \varphi_{2} \chi_{2}^{\dagger}, \quad \psi_{1}^{\dagger} \phi_{1} \varphi_{1} \chi_{2}+\psi_{2}^{\dagger} \phi_{2} \varphi_{2} \chi_{1}, \\
& \psi_{1} \phi_{1}^{\dagger} \varphi_{1} \chi_{2}+\psi_{2} \phi_{2}^{\dagger} \varphi_{2} \chi_{1}, \quad \psi_{1} \phi_{1} \varphi_{1}^{\dagger} \chi_{2}+\psi_{2} \phi_{2} \varphi_{2}^{\dagger} \chi_{1}, \\
& \psi_{1} \phi_{2} \varphi_{2} \chi_{2}^{\dagger}+\psi_{2} \phi_{1} \varphi_{1} \chi_{1}^{\dagger}, \quad \psi_{1}^{\dagger} \phi_{2} \varphi_{1} \chi_{1}+\psi_{2}^{\dagger} \phi_{1} \varphi_{2} \chi_{2}, \\
& \psi_{1}^{\dagger} \phi_{1} \varphi_{2} \chi_{1}+\psi_{2}^{\dagger} \phi_{2} \varphi_{1} \chi_{2}, \quad \psi_{1} \phi_{1} \varphi_{2} \chi_{2}+\psi_{2} \phi_{2} \varphi_{1} \chi_{1}, \\
& \psi_{1} \phi_{2} \varphi_{1} \chi_{2}+\psi_{2} \phi_{1} \varphi_{2} \chi_{1}, \quad \psi_{1} \phi_{2} \varphi_{2} \chi_{1}+\psi_{2} \phi_{1} \varphi_{1} \chi_{2} .
\end{array}
$$

Their hermitian conjugates are also in the $1_{S}$ representations. 


\subsection{Group basis dependence}

We have presented the $S_{3}$ algebra in the complex basis where the representation matrices are given by those in Table 1 . There are, however, several bases of the $S_{3}$ matrices which are often used in the literature. So it may be instructive here to describe the relation among these group bases, compared to the complex basis used in the previous subsections.

\subsubsection{Democratic basis}

The democratic basis is adopted to produce a flavor-democratic mass matrix in which all matrix elements are equal [14]. In this basis, the $S_{3}$ operations generate the permutations of three objects, for example, the exchange of the first and second indices. The invariance under such transformations require the universal size of couplings for three generations if they belong to a three-dimensional representation of $S_{3}$ and the Higgs field is in the singlet.

Unlike in the complex basis, the representation matrices have apparently non-trivial (not block-diagonal) structure for three-dimensional representations. Different group bases are converted to each other by unitary transformations. It is worth noting that there are two types of democratic basis which correspond to the existence of two threedimensional representations; $3_{\mathrm{S}}$ and $3_{\mathrm{A}}$. The elements in the democratic basis for $3_{\mathrm{S}}$ are defined by the unitary matrix $V$ as

$$
T_{i}^{S}=V T_{i} V^{\dagger},
$$

where $T_{i}$ 's in the right-handed side are given in the complex basis, and $V \equiv U P_{S}$ with

$$
U=\left(\begin{array}{ccc}
1 / \sqrt{2} & 1 / \sqrt{6} & 1 / \sqrt{3} \\
-1 / \sqrt{2} & 1 / \sqrt{6} & 1 / \sqrt{3} \\
0 & -2 / \sqrt{6} & 1 / \sqrt{3}
\end{array}\right), \quad P_{S}=\left(\begin{array}{ccc}
1 / \sqrt{2} & -1 / \sqrt{2} & \\
i / \sqrt{2} & i / \sqrt{2} & \\
& & 1
\end{array}\right) .
$$

Thus the representation matrices for $3_{\mathrm{S}}$ are given by the label-changing matrices:

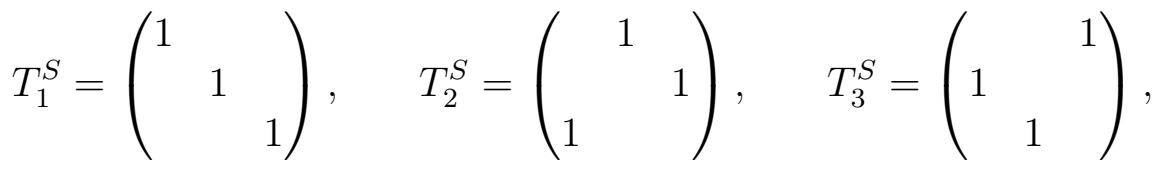

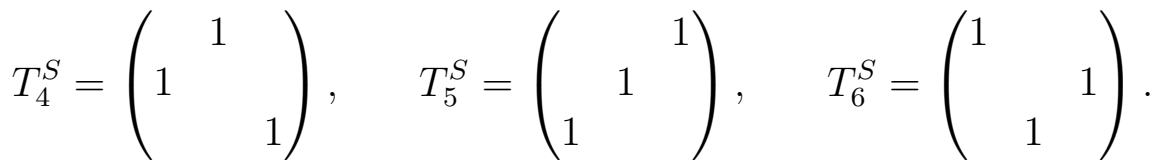

For the pseudo-triplet representation $3_{\mathrm{A}}$, the matrices $T_{1,2,3}^{S}$ have the same forms as above, but $T_{4,5,6}^{S}$ become rather complicated, as can be seen by the definition (2.12). 
Another democratic basis is defined so that the representation matrices for $3_{\mathrm{A}}$ are expressed by label-exchanging matrices like (2.14). The matrices $T_{i}^{A}$ are given by a similar unitary rotation to (2.12), except that the matrix $P_{S}$ is now replaced by

$$
P_{A}=\left(\begin{array}{ccc}
1 / \sqrt{2} & 1 / \sqrt{2} & \\
i / \sqrt{2} & -i / \sqrt{2} & \\
& & 1
\end{array}\right) .
$$

One can see that the matrices $T_{1,2,3}^{A}$ take the same forms as those in (2.14) both for triplet and pseudo-triplet representations. The differences appear for $T_{4,5,6} ; T_{4,5,6}^{A}\left(3_{\mathrm{A}}\right)=$ $-T_{4,5,6}^{S}\left(3_{\mathrm{S}}\right)$ and $T_{4,5,6}^{A}\left(3_{\mathrm{S}}\right)=-T_{4,5,6}^{S}\left(3_{\mathrm{A}}\right)$, the former matrices exchanges the three objects as (2.14) and the latter have some complicated forms.

\subsubsection{Real basis}

In the discussion of the democratic basis, $P_{S, A}$ rotate only the first and second indices. This means that the representation matrices $T_{i}^{S, A}$ are rotated to block-diagonal forms only by the $U$ rotation, namely, the $U$-rotated matrices are decomposed into the matrices for irreducible representations. (Note that $U$ is the unitary rotation which diagonalizes the so-called flavor-democratic mass matrix.) The real basis $T_{i}^{U}$ is defined by rotating $T_{i}^{S, A}$ with the $U$ matrix, for example,

$$
T_{i}^{U}=U^{\dagger} T_{i}^{S} U
$$

These group elements take the following forms for the $3_{\mathrm{S}}$ representation:

$$
\begin{aligned}
& T_{1}^{U}=\left(\begin{array}{lll}
1 & & \\
& 1 & \\
& & 1
\end{array}\right), \quad T_{2}^{U}=\left(\begin{array}{ccc}
-1 / 2 & \sqrt{3} / 2 & \\
-\sqrt{3} / 2 & -1 / 2 & \\
& & 1
\end{array}\right), \quad T_{3}^{U}=\left(\begin{array}{ccc}
-1 / 2 & -\sqrt{3} / 2 & \\
\sqrt{3} / 2 & -1 / 2 & \\
& & 1
\end{array}\right), \\
& T_{4}^{U}=\left(\begin{array}{lll}
-1 & & \\
& 1 & \\
& & 1
\end{array}\right), \quad T_{5}^{U}=\left(\begin{array}{ccc}
1 / 2 & -\sqrt{3} / 2 & \\
-\sqrt{3} / 2 & -1 / 2 & \\
& & 1
\end{array}\right), \quad T_{6}^{U}=\left(\begin{array}{ccc}
1 / 2 & \sqrt{3} / 2 & \\
\sqrt{3} / 2 & -1 / 2 & \\
& & 1
\end{array}\right),
\end{aligned}
$$

which are block diagonal. It is clear from the definition that the $T^{U}$ basis is obtained from the complex basis by the $P_{S}$ or $P_{A}$ phase rotation. Note that, unlike in the complex basis, all the representation matrices are real-valued. This is why we call it the real basis. Due to this reality of matrix elements of $T_{i}^{U}$, two types of tensor products are possible for $S_{3}$ doublets. In the real basis of (2.16), the one tensor product of two doublets is

$$
\begin{aligned}
\psi \times \phi & =\left(\psi^{\dagger} \sigma_{1} \phi, \psi^{\dagger} \sigma_{3} \phi\right)_{2}^{\mathrm{t}}+\left(\psi^{\dagger} i \sigma_{2} \phi\right)_{1_{\mathrm{A}}}+\left(\psi^{\dagger} \phi\right)_{1_{\mathrm{S}}} \\
& =\left(\psi_{1}^{\dagger} \phi_{2}+\psi_{2}^{\dagger} \phi_{1}, \psi_{1}^{\dagger} \phi_{1}-\psi_{2}^{\dagger} \phi_{2}\right)_{2}^{\mathrm{t}}+\left(\psi_{1}^{\dagger} \phi_{2}-\psi_{2}^{\dagger} \phi_{1}\right)_{1_{\mathrm{A}}}+\left(\psi_{1}^{\dagger} \phi_{1}+\psi_{2}^{\dagger} \phi_{2}\right)_{1_{\mathrm{S}}} .
\end{aligned}
$$


Another consistent product can be defined by using transposition of $\psi$, instead of $\psi^{\dagger}$. This choice is possible since all the group elements are expressed in terms of real numbers. In the language of the complex basis, the product defined with daggers corresponds to (2.4) and that with transpositions to (2.7). The difference between these two types of products becomes important in the case that $S_{3}$-doublet fields transform non-trivially under other symmetries than $S_{3}$. In particular, this is indeed the case for the standardmodel fermions and Higgs bosons. The real basis has often been used in the literature [15]. For completeness, we comment on another real basis which is defined from $T_{i}^{A}$, instead of $T_{i}^{S}$ in (2.16). The matrices $T_{i}^{U}$ are now given by $T_{i}^{U}=U^{\dagger} T_{i}^{A} U$ and their explicit forms for $3_{\mathrm{A}}$ are (2.17) by changing the signs of $T_{4,5,6}^{U}$ (i.e., $T_{4,5,6}^{U} \rightarrow-T_{4,5,6}^{U}$ ). Therefore the non-trivial tensor product becomes $\psi \times \phi=\left(\psi^{\dagger} \sigma_{3} \phi,-\psi^{\dagger} \sigma_{1} \phi\right)_{2}^{\mathrm{t}}+\left(\psi^{\dagger} i \sigma_{2} \phi\right)_{1_{\mathrm{A}}}+\left(\psi^{\dagger} \phi\right)_{1_{\mathrm{S}}}$ or that with $\psi^{\mathrm{t}}$.

Finally we show the singlet components contained in the products of more than two doublets. In the real basis, they are given by (up to the fourth order)

$$
\begin{aligned}
& \psi \times \phi \quad \supset \psi_{1} \phi_{1}+\psi_{2} \phi_{2}, \\
& \psi \times \phi \times \varphi>\psi_{1} \phi_{1} \varphi_{2}+\psi_{1} \phi_{2} \varphi_{1}+\psi_{2} \phi_{1} \varphi_{1}-\psi_{2} \phi_{2} \varphi_{2} \text {, } \\
& \psi \times \phi \times \varphi \times \chi \supset\left(\psi_{1} \phi_{1}+\psi_{2} \phi_{2}\right)\left(\varphi_{1} \chi_{1}+\varphi_{2} \chi_{2}\right), \quad\left(\psi_{1} \varphi_{1}+\psi_{2} \varphi_{2}\right)\left(\phi_{1} \chi_{1}+\phi_{2} \chi_{2}\right), \\
& \left(\psi_{1} \chi_{1}+\psi_{2} \chi_{2}\right)\left(\phi_{1} \varphi_{1}+\phi_{2} \varphi_{2}\right), \quad\left(\psi_{1} \phi_{2}-\psi_{2} \phi_{1}\right)\left(\varphi_{1} \chi_{2}-\varphi_{2} \chi_{1}\right), \\
& \left(\psi_{1} \varphi_{2}-\psi_{2} \varphi_{1}\right)\left(\phi_{1} \chi_{2}-\phi_{2} \chi_{1}\right), \quad\left(\psi_{1} \chi_{2}-\psi_{2} \chi_{1}\right)\left(\phi_{1} \varphi_{2}-\phi_{2} \varphi_{1}\right) \text {. }
\end{aligned}
$$

In the right-handed sides, the components $\psi_{i}, \phi_{j}, \cdots$ can be replaced with $\phi_{i}^{\dagger}, \phi_{j}^{\dagger}, \cdots$. Namely, $\left(\psi_{1}^{\dagger} \phi_{1}+\psi_{2}^{\dagger} \phi_{2}\right),\left(\psi_{1} \phi_{1}^{\dagger} \varphi_{2}+\psi_{1} \phi_{2}^{\dagger} \varphi_{1}+\psi_{2} \phi_{1}^{\dagger} \varphi_{1}-\psi_{2} \phi_{2}^{\dagger} \varphi_{2}\right)$, and others are also $S_{3}$ singlets. This is due to the fact that the representation matrices $T_{i}^{U}$ are real in the real basis.

\section{Invariant matrices}

Various forms of mass textures of quarks and leptons have been known to be phenomenologically viable [1]-[5]. As discussed in the previous section, $S_{3}$ has three types of irreducible representations; $2,1_{\mathrm{A}}$ and $1_{\mathrm{S}}$. We would like here to examine what combinations of $S_{3}$ representations for matter fields lead to those mass textures. Higgs fields may also transform non-trivially under the $S_{3}$ symmetry and play significant roles for realizing textures forms. Since there are three repetitions of matter fields, various assignments of $S_{3}$ representations are available, where the first two generations constitute a doublet and so on. These include a possibility to realize asymmetrical forms of mass textures and give rise 
to physically observable effects such as flavor-violating processes in future experiments. In particular, as mentioned in the introduction, vanishing matrix elements lead to interesting phenomenological consequences. Such zero elements could be obtained in the framework of non-abelian discrete flavor symmetry by suitably taking Higgs representations and their VEV forms, which are calculable by analyzing scalar potential. Natural forms of VEVs generally depend on the $S_{3}$ group basis in which mass matrices are described. At this stage, explicit forms of mass textures are governed only by discrete flavor symmetry. In some cases, however, additional implementation might be needed to have more control, in particular, for fermion mass hierarchy being naturally realized. In the present framework, the key ingredients for model construction are to select (i) $S_{3}$ group structure, (ii) representations of matter fields, (iii) Higgs profiles (representations and VEVs), and (iv) extra symmetries. Let us first comment on these issues in some details.

(i) $S_{3}$ group structures: As mentioned in Section 2.3, physical consequences of a mass texture potentially depend on the group basis adopted in constructing $S_{3}$ models. It is noted that a choice of specific basis does not affect physical results as long as the flavor symmetry is unbroken: apparent basis dependence, e.g. different forms of mass matrices, is only due to a choice of flavor basis. The $S_{3}$ invariance guarantees the same spectrum without regard to basis choices. However the predictions for generation mixing might be different. This is because, in the standard model, there already exists a basis which defines the generation structure, namely the interaction basis where the weak current interaction is flavor diagonal. Once one picks up a group basis from some model-building perspective, the relabeling of flavor indices by $S_{3}$ transformation gives physical meaning on generation structure. Another important basis dependence appears in breaking the flavor symmetry. Since any kind of flavor symmetry has not been observed in the lowenergy regime, in principle any form of symmetry-breaking patterns is possible. In realistic model construction, some guiding principles are often adopted, such as simplicity and/or dynamical justification. In either case, symmetry-breaking parameters depend on the $S_{3}$ basis. While the breaking parameters take a simple form in one basis, they are rotated to a complicated form in other general bases, which form seems to be completely unnatural. In this way, the choice of flavor-group basis may have physical consequences if threegeneration fermions are assigned to (pseudo) triplets, and therefore is an important factor in constructing models with flavor symmetry.

Another issue is what types of $S_{3}$ symmetries are involved into the theory. For example, with only one $S_{3}$ symmetry, both left- and right-handed fermions (and also Higgs bosons) transform under the same $S_{3}$. On the other hand, one may easily imagine that threegeneration fermions have non-trivial charges of separate $S_{3}$ groups. A well-known example of the latter case is the democratic mass texture [14] realized by $S_{3 L} \times S_{3 R}$ symmetries. 
(ii) Representations of matter fields: In this paper, $S_{3}$ is introduced to control the flavor structure of quarks and leptons. Three-generation matter fields generally belong to non-trivial representations of such flavor symmetries. A charge assignment often assumed in the literature is that the first and second generations make up a doublet. This assignment is adopted to account for several phenomenological issues. First, the flavor symmetry invariance suppresses flavor-violating effects between the first and second generations, which effects have been tightly constrained by various experimental results in supersymmetric theory [17]. Second, if the third generation has different flavor charges from the other twos, they are appropriate to have larger masses. It is however noted that all other flavor charge assignments are equally allowed. For example, a phenomenologically interesting alternative is that the second and third generation leptons make an $S_{3}$ doublet. This is motivated by the recent observations of neutrino flavor mixing between the second and third generations. Moreover it could account for the lightness of the first-generation fermions. In what follows, we show that representations of matter fields, including left-right asymmetric assignments, are useful for obtaining various types of mass matrices.

(iii) Higgs profiles: Phenomenologically indirect but important possibilities arise for the profile of Higgs fields. If $S U(2)_{W}$-doublet Higgses are in non-trivial representations of $S_{3}$, Yukawa couplings can be described by renormalizable operators. Higher-dimensional operators including multiple Higgs fields are suppressed by a large cutoff scale and give negligible corrections to Yukawa couplings, which is a nice feature in a sense that everything is described within the renormalizable level. This approach however requires that hierarchically small values of Yukawa couplings for the first two generations must be fixed by hand as in the standard model. Moreover, due to the existence of multiple $S U(2)_{W}$-doublet Higgses, naive gauge coupling unification is spoiled, and Higgs-mediated flavor-violating effects might not be negligible even at tree level. An alternative choice of Higgs charges is that $S U(2)_{W}$-doublet Higgses belong to the flavor singlet. In this case, Yukawa interactions are effectively derived from higher-dimensional operators which can be made invariant by introducing appropriate scalars $\Phi$ 's in non-trivial $S_{3}$ representations. The VEVs of $\Phi$ break the flavor symmetry and generate trilinear Yukawa couplings below the breaking scale. Since the ratio $\langle\Phi\rangle$ to a cutoff scale gives a unit of Yukawa hierarchy of quarks and leptons, $S_{3}$ should be broken at a high-energy scale below the cutoff. This situation resolves the above-mentioned problems of $S_{3}$-charged $S U(2)_{W}$ Higgs doublets: Yukawa hierarchy is explained by controlled higher-dimensional operators, gauge coupling unification is preserved, and Higgs-mediated flavor violation is suppressed by a large $S_{3}$-breaking scale. As an imprint of such high-scale flavor symmetry, new sources of flavor violation could arise from renormalization-group evolution below the symmetry- 
breaking scale. For example, if the theory is supersymmetrized, flavor-changing couplings are generally induced, and their magnitudes depend on the dynamics of supersymmetry breaking. While the flavor violation tends to be small as Kähler terms are limited by flavor symmetry, it may be observable, e.g. in the gravity mediation scenario [18]. Note that this type of flavor violation is negligible in the case of $S_{3}$-charged Higgs bosons as long as the flavor symmetry remains intact at low-energy regime.

The $S_{3}$ property of Higgs fields is also relevant to the group basis. That is the form of VEVs and their naturalness in the sense of 't Hooft. For example, if a pseudo-singlet field develops a non-vanishing VEV,$S_{3}$ is broken down to a subgroup $Z_{3}$. In the limit of other VEVs being zeros, low-energy effective theory still has the residual $Z_{3}$ invariance. In fact, for realistic cases, some $S_{3}$ doublets have nonzero VEVs in order to give non-trivial flavor structure. Then the maximal residual subgroup is $S_{2}$. Notice here that the $S_{2}$-invariant forms of VEVs depend on the group basis. It is found from (2.3) and (2.17) that such technically natural VEV of $S_{3}$ doublet is proportional to $(1,1)^{\mathrm{t}}$ in the complex basis and $(0,1)^{\mathrm{t}}\left[\right.$ or $\left.(1,0)^{\mathrm{t}}\right]$ in the real basis. The former is available to realize large flavor mixing and the latter is useful for generating textures with vanishing elements. Since the flavor symmetry is completely broken at low energy, the group basis might be chosen so that symmetry-breaking forms seem as natural as possible. Finally, there is another form of doublet VEV which is often utilized in the literature. That is $(x, 1)^{\mathrm{t}}$ with $x \ll 1$, which arises from a linear combination of the above two types of VEVs, but it is nothing but parameter tuning.

(iv) Extra symmetries: The $S_{3}$ flavor symmetry does not fully explain the mass hierarchies of quarks and leptons. A hierarchical order of $S_{3}$-breaking VEVs requires different order of couplings whose origin is generally unclear. An attractive way to dynamically justify hierarchical couplings is to introduce extra symmetries into the theory. As for Yukawa couplings, a well-known example is the Froggatt-Nielsen mechanism [19] where extra symmetry controls the orders of Yukawa couplings. They are generated in low-energy effective theory via decoupling heavy fields, i.e. higher-dimensional operators whose coefficients are naturally given by the fundamental scale of the theory.

In the following, we present several examples of mass matrices which stem from the $S_{3}$ flavor symmetry. In almost the cases, three-generation fermions belong to non-trivial triplet representations: two of them make up an $S_{3}$ doublet and the other is a (pseudo) singlet. On the other hand, appropriate representations of Higgs fields are chosen to have non-vanishing elements in $S_{3}$-invariant Yukawa matrices. Mass textures will be expressed in the complex basis unless particularly mentioned. The expressions in the other bases are easily obtained by the unitary basis rotations defined in the previous section. 


\subsection{A single $S_{3}$}

First we study the case that both left-handed fermions $\psi_{L i}$ and right-handed ones $\psi_{R j}$ $(i, j=1,2,3)$ transform under a single $S_{3}$ group. This case is also straightforwardly applied to Majorana mass terms with the identification $\psi_{L}=\psi_{R}$. In the following, we take a charge assignment that $\left(\psi_{L 1}, \psi_{L 2}\right)$ and $\left(\psi_{R 1}, \psi_{R 2}\right)$ are $S_{3}$ doublets and the other fermions are in (pseudo) singlet representation. This does not lose any generalities since mass matrices for other charge assignments are obtained by flavor rotations. As for Higgs fields, all the three types of irreducible representations are available for giving nonzero matrix elements; a doublet $H_{D}=\left(H_{1}, H_{2}\right)$, a pseudo singlet $H_{A}$, and a singlet $H_{S}$. Here $H_{D, S, A}$ are interpreted as either $S U(2)_{W}$-doublet elementary scalars or products of some numbers of fields [see, e.g. the comment (iii) Higgs profiles in the above].

\subsubsection{The general case}

Dirac mass terms flip the chirality of fermions. Given that $\psi_{L 3}$ and $\psi_{R 3}$ are singlets, the most general $S_{3}$-invariant Dirac mass matrix is described as

$$
M=\left(\begin{array}{ccc}
\mathcal{L}_{\text {Dirac }}=-\overline{\psi_{R i}} M_{i j} \psi_{L j}+\text { h.c. } & \\
c H_{S}+a^{\prime} H_{S}^{*}+b H_{A}+b^{\prime} H_{A}^{*} & c H_{2}+c^{\prime} H_{1}^{*} & d H_{1}+e^{\prime} H_{2}^{*} \\
c H_{1}+c^{\prime} H_{2}^{*} & a H_{S}+a^{\prime} H_{S}^{*}-b H_{A}-b^{\prime} H_{A}^{*} & d H_{2}+e^{\prime} H_{1}^{*} \\
e H_{2}+d^{\prime} H_{1}^{*} & e H_{1}+d^{\prime} H_{2}^{*} & f H_{S}+f^{\prime} H_{S}^{*}
\end{array}\right),
$$

where $a, a^{\prime}, b, \cdots, f, f^{\prime}$ are independent coupling constants. The generic form of mass matrix (3.2) is simplified if $H_{D, S, A}$ belong to complex representations of other groups than $S_{3}$ : either $H_{x}$ or corresponding $H_{x}^{*}$ is dropped out in each element of the generic matrix. This is indeed the case for the $S U(2)_{W}$ Higgs doublets in the standard model. Another charge assignment is that the pseudo singlet representation $1_{\mathrm{A}}$ is adopted for the third-generation fermions. For example, when $\psi_{L 3}$ is a pseudo singlet, the third column of (3.2) is modified so that $H_{2}$ and $H_{S}$ are replaced with $-H_{2}$ and $H_{A}$, respectively.

The general form of Majorana mass matrix is described by identifying $\psi_{L}=\psi_{R} \equiv \psi$. It is noted that the tensor product (2.7) should be used in constructing Majorana mass term in the complex basis. Thus the $S_{3}$ invariance leads to

$$
\begin{gathered}
\mathcal{L}_{\text {Majorana }}=-\frac{1}{2} \overline{\psi_{i}^{c}} M_{i j} \psi_{j}+\text { h.c. } \\
M=\left(\begin{array}{ccc}
a H_{1}+b H_{2}^{*} & c H_{S}+c^{\prime} H_{S}^{*} & d H_{2}+e H_{1}^{*} \\
c H_{S}+c^{\prime} H_{S}^{*} & a H_{2}+b H_{1}^{*} & d H_{1}+e H_{2}^{*} \\
d H_{2}+e H_{1}^{*} & d H_{1}+e H_{2}^{*} & f H_{S}+f^{\prime} H_{S}^{*}
\end{array}\right) .
\end{gathered}
$$

Similar to the above case of Dirac masses, either $H_{x}$ or $H_{x}^{*}$ is removed in each matrix element when $H_{x}$ has some (complex) quantum charge other than that of $S_{3}$. Due to 
the difference of $S_{3}$ tensor products used for Dirac and Majorana mass terms, the resulting flavor structures in (3.2) and (3.4) are rather different. This fact could provide an interesting possibility for realistic model construction of flavor.

\subsubsection{Supersymmetric case}

Supersymmetry invariant fermion masses come from the superpotential, which is a holomorphic function of chiral superfields. That is, superpotential terms are written in terms of chiral superfields, which contain fermionic components with a certain chirality, e.g. left-handed fermions. In this case, right-handed fermions are introduced as charge conjugations of left-handed ones. As in the general case, suppose that the chiral superfields of first two generations, $\left(\Psi_{L 1}, \Psi_{L 2}\right)$ and $\left(\Psi_{R 1}, \Psi_{R 2}\right)$, are $S_{3}$ doublets, and $\Psi_{R 3}, \Psi_{L 3}$ are singlets. It should be noted that this assignment leads to the right-handed fermions $\left(\psi_{R 1}, \psi_{R 2}\right)$ being an anti-doublet. Therefore the product (2.7) is applied to constructing $S_{3}$-invariant superpotential. The most general supersymmetric mass term is thus given by

$$
\begin{gathered}
W_{\text {Dirac }}=\Psi_{R i} M_{i j} \Psi_{L j}, \\
M=\left(\begin{array}{ccc}
a H_{1} & b H_{S}+c H_{A} & d H_{2} \\
b H_{S}-c H_{A} & a H_{2} & d H_{1} \\
e H_{2} & e H_{1} & f H_{S}
\end{array}\right) .
\end{gathered}
$$

In the case that $\Psi_{L 3}$ is assigned to a pseudo singlet, one needs the replacement $H_{2} \rightarrow-H_{2}$ and $H_{S} \rightarrow H_{A}$ in the third column of (3.6). Similarly, the $S_{3}$-invariant Majorana mass term is easily found by symmetrizing the matrix (3.6), namely, by setting the couplings as $c=0$ and $d=e$. This does not significantly modify the flavor structure, unlike in the non-supersymmetric case.

\subsubsection{Relation to the democratic mass matrix}

We have descried the mass matrices in the complex basis of the $S_{3}$ group. Let us here comment on the relation to the so-called democratic form of mass matrix in which all the matrix elements have equal magnitude. It has a simple $S_{3}$ derivation in the democratic basis defined in Section 2.3.1. As we explained before, these two group bases are connected by the unitary rotation $U$ in (2.13). A simple way to recover the democratic mass matrix is to introduce only an $S_{3}$-singlet Higgs field $H_{S}$. As seen from (3.2), that corresponds to a flavor-diagonal matrix in the complex basis. By rotating the matrix to the democratic 
basis, it turns out to be

$$
M=\left(\begin{array}{lll}
1 & 1 & 1 \\
1 & 1 & 1 \\
1 & 1 & 1
\end{array}\right) h_{S}+\left(\begin{array}{lll}
1 & & \\
& 1 & \\
& & 1
\end{array}\right) h_{S}^{\prime}
$$

where $h_{S}=\left[(f-a) H_{S}+\left(f^{\prime}-a^{\prime}\right) H_{S}^{*}\right] / 3$ and $h_{S}^{\prime}=a H_{S}+a^{\prime} H_{S}^{*}$. It is not hard to see the $S_{3}$ invariance of the above two matrices in the democratic basis. The democratic mass matrix is thus found to be derived from an assumption that only one matrix element is dominant in the complex basis. Therefore a single $S_{3}$ symmetry cannot ensure the flavor democracy: even with a single $H_{S}$ field, the democratic ansatz is generally disturbed. As seen from the representation matrices (2.14), the democratic basis deals with the three indices of a (pseudo) triplet equivalently, and is not suitable to discuss the decomposition to irreducible representations.

\section{$3.2 S_{3 L} \times S_{3 R}$}

For controlling chirality-flipping operators, one can utilize a flavor symmetry under which left-handed and right-handed fermions transform separately, that is, the $S_{3 L} \times S_{3 R}$ symmetry. Without loss of any generalities, we assume that the first two generations $\left(\psi_{L 1}, \psi_{L 2}\right)$ and $\left(\psi_{R 1}, \psi_{R 2}\right)$ belong to the doublet representations of $S_{3 L}$ and $S_{3 R}$, respectively. Since there exist three irreducible representations for the $S_{3}$ group, fermion bilinear terms transform in nine ways under $S_{3 L} \times S_{3 R}$. The corresponding nine types of scalars which ensures the flavor invariance are denoted by $H_{i j}(i, j=D, S, A)$, where an obvious notation has been used, e.g. $H_{D A}$ means a doublet under $S_{3 L}$ and a pseudo singlet of $S_{3 R}$. As mentioned before, the symbols $H_{i j}$ stand for either $S U(2)_{W}$-doublet elementary scalars or products of some fields with appropriate charges.

\subsubsection{The general case}

For an illustration, we assume that the third-generation fermions $\psi_{L 3}$ and $\psi_{R 3}$ are singlets of $S_{3 L}$ and $S_{3 R}$, respectively. In case that they are pseudo singlets, the subscripts $S$ of Higgs fields should be replaced with $A$ in the following expressions. The most general Dirac mass matrix is made symmetry invariant by introducing $H_{D D}, H_{D S}, H_{S D}$, and 
$H_{S S}$, and is given by

$$
\begin{gathered}
\mathcal{L}_{\text {Dirac }}=-\overline{\psi_{R i}} M_{i j} \psi_{L j}+\text { h.c. } \\
M=\left(\begin{array}{ccc}
a\left(H_{D D}\right)_{11}+b\left(H_{D D}\right)_{12} & a\left(H_{D D}\right)_{12}+b\left(H_{D D}\right)_{11} & \\
+c\left(H_{D D}^{*}\right)_{21}+d\left(H_{D D}^{*}\right)_{22} & +c\left(H_{D D}^{*}\right)_{22}+d\left(H_{D D}^{*}\right)_{21} & \\
a\left(H_{D D}\right)_{21}+b\left(H_{D D}\right)_{22} & a\left(H_{D D}\right)_{22}+b\left(H_{D D}\right)_{21} & \\
+c\left(H_{D D}^{*}\right)_{11}+d\left(H_{D D}^{*}\right)_{12} & +c\left(H_{D D}^{*}\right)_{12}+d\left(H_{D D}^{*}\right)_{11} & \\
g\left(H_{D S}\right)_{2}+h\left(H_{D S}^{*}\right)_{1} & g\left(H_{D S}\right)_{1}+h\left(H_{D S}^{*}\right)_{2} & \left.j H_{S S}+j^{\prime} H_{S S}^{*}\right)_{1}
\end{array}\right),
\end{gathered}
$$

where $a, b, \cdots, j, j^{\prime}$ are the coupling constants. The Higgs fields either with or without asterisks are dropped out in each matrix element if they have some quantum numbers of other symmetries than $S_{3 L} \times S_{3 R}$. The possible Majorana mass term of $\psi_{L}$ (or $\left.\psi_{R}\right)$ is written in the same way as (3.4) by including other $H$ 's with appropriate charges.

The Dirac mass term in supersymmetric theory is given by the superpotential which is a analytic function of superfields with definite chirality. If we take the same flavor charge assignment as above for the three-generation superfields $\Psi_{L i}$ and $\Psi_{R j}$, the most general form of mass matrix is

$$
\begin{gathered}
W_{\text {Dirac }}=\Psi_{R i} M_{i j} \Psi_{L j} \\
M=\left(\begin{array}{ccc}
a\left(H_{D D}\right)_{22} & a\left(H_{D D}\right)_{21} & b\left(H_{S D}\right)_{2} \\
a\left(H_{D D}\right)_{12} & a\left(H_{D D}\right)_{11} & b\left(H_{S D}\right)_{1} \\
c\left(H_{D S}\right)_{2} & c\left(H_{D S}\right)_{1} & d H_{S S}
\end{array}\right) .
\end{gathered}
$$

\subsubsection{Examples}

It is found from (3.9) that the general form of Dirac mass term is rather complicated. Combining with simple assumptions, we here present several examples where phenomenologically interesting forms of mass matrices are obtained, in particular, by choosing relevant Higgs contents.

The first example is the introduction of a single elementary scalar $H$ in the (pseudo) singlet representation of both $S_{3 L}$ and $S_{3 R}$ symmetries. According to the $S_{3}$ charges of $\psi_{L 3, R 3}$, a relevant representation of the scalar becomes that of $H_{i j}$ with $i, j=S$ or $A$. In any case, only the $3-3$ element of mass matrix is allowed;

$$
M=\left(\begin{array}{ll} 
& \\
& a H
\end{array}\right) .
$$


If turning to the democratic basis, we have

$$
M=\frac{1}{3}\left(\begin{array}{lll}
1 & 1 & 1 \\
1 & 1 & 1 \\
1 & 1 & 1
\end{array}\right) a H
$$

Both of these matrices have only one nonzero eigenvalue and the same mass spectrum. However the resulting flavor mixing structures are clearly different: the matrix (3.13) induces large flavor mixing, while (3.12) has no mixing between the third and the firsttwo generations. As we noted before, this may be a physical difference because, in realistic models, there exists more than one sector whose relative basis differences do affect flavor structure such as the quark mixing angles.

The second example is not to consider that elementary scalars $H_{i j}$ give all trilinear Yukawa couplings, but to work with some products of scalars in lower-dimensional representations. As an example, let us introduce three scalar fields $H_{D}=\left(H_{1}, H_{2}\right)$, $H_{D}^{\prime}=\left(H_{1}^{\prime}, H_{2}^{\prime}\right)$ and $H_{S}$ whose representations of $\left(S_{3 L}, S_{3 R}\right)$ are $\left(2,1_{\mathrm{S}}\right),\left(1_{\mathrm{S}}, 2\right)$, and $\left(1_{\mathrm{S}}, 1_{\mathrm{S}}\right)$, respectively. With this field content and the matter representation as above, all entries in the Dirac mass matrix can be filled up with non-renormalizable operators. In supersymmetric theory, the mass matrix is now given by

$$
M=\left(\begin{array}{ccc}
a H_{2} H_{2}^{\prime} & a H_{1} H_{2}^{\prime} & b H_{2}^{\prime} \\
a H_{2} H_{1}^{\prime} & a H_{1} H_{1}^{\prime} & b H_{1}^{\prime} \\
c H_{2} & c H_{1} & d H_{S}
\end{array}\right)
$$

On top of economical field content, this example has several useful properties for constructing flavor models of quarks and leptons. First, the matrix (3.14) has a vanishing determinant and therefore provides a compelling dynamical reason for the observed tiny masses of first-generation fermions. Secondly, the effective Yukawa couplings for the first-two generations become naturally small. This is because the $S_{3}$ invariance requires that they come from higher-dimensional operators suppressed by some large mass scale. Thirdly, the hierarchical flavor structure is easily attained with a smaller number of scalar VEVs. It might often lead to some relations among mass eigenvalues and mixing angles of quarks and leptons. One can make use of these interesting features with a smaller number of representations of scalar fields than (3.9).

\subsection{Singlet flavor}

We have so far discussed the case that both left- and right-handed fermions have nontrivial charges of discrete flavor symmetry. In this subsection, we comment on a possibility that either left- or right-handed generations is insensitive to flavor transformation. 
Let us consider a single $S_{3}$ symmetry under which left-handed generations transform non-trivially but right-handed ones do not. In a similar way to the discussion in Section 3.1.1. the generic types of representations, $H_{D}=\left(H_{1}, H_{2}\right), H_{S}$, and $H_{A}$, are taken into account. If one takes a charge assignment that $\left(\psi_{L 1}, \psi_{L 2}\right)$ is a doublet, the most general Dirac mass matrix is given by

$$
\begin{gathered}
\mathcal{L}_{\text {Dirac }}=-\overline{\psi_{R i}} M_{i j} \psi_{L j}+\text { h.c. } \\
M=\left(\begin{array}{ccc}
a H_{2}+b H_{1}^{*} & a H_{1}+b H_{2}^{*} & g H_{S}+g^{\prime} H_{S}^{*} \\
c H_{2}+d H_{1}^{*} & c H_{1}+d H_{2}^{*} & h H_{S}+h^{\prime} H_{S}^{*} \\
e H_{2}+f H_{1}^{*} & e H_{1}+f H_{2}^{*} & j H_{S}+j^{\prime} H_{S}^{*}
\end{array}\right) .
\end{gathered}
$$

In each matrix element, a symbol $H$ either with or without asterisk should be dropped if $H$ is accompanied with some charges other than that of $S_{3}$. In supersymmetric theory, the mass matrix is simplified to

$$
M=\left(\begin{array}{ccc}
a H_{2} & a H_{1} & g H_{S} \\
c H_{2} & c H_{1} & h H_{S} \\
e H_{2} & e H_{1} & j H_{S}
\end{array}\right) .
$$

If only a singlet Higgs field exists, that results in

$$
\text { (3.17) } \rightarrow\left(\begin{array}{ccc}
g & g & g \\
h & h & h \\
j & j & j
\end{array}\right) H_{S}
$$

in the democratic basis. This form of Dirac mass matrix has been discussed in the so-called lopsided models. Similar results are also obtained with the $1_{\mathrm{A}}$ representation instead of $1_{\mathrm{S}}$.

\section{Mass textures from $S_{3}$}

On the prescription described in the previous sections, we will perform several constructions of mass textures by use of flavor $S_{3}$ symmetry. That includes well-established forms of mass matrices which have been discussed in the literature. In realistic flavor models for quarks and leptons, some combinations of texture forms are usually assumed, and it is therefore meaningful to examine whether they can be simultaneously reproduced by horizontal symmetry.

It is found in Section 3 that the key ingredients for $S_{3}$ model building are to define matter and Higgs profiles and, if needed, to introduce additional symmetry. Among such further symmetries, we will focus in this work on $U(1)$ flavor symmetry to dynamically 
realize fermion mass hierarchy [19, 20]. It is, however, highly non-trivial to assign relevant $U(1)$ quantum numbers. This is because an $S_{3}$ doublet, e.g. the first and second generations in the above matrix examples, must have the same $U(1)$ charge and it does not provide mass hierarchies between them. Moreover, a naive charge assignment often leads to a prediction that classical mass hierarchy realized by flavor symmetry is disturbed by higher-dimensional operators. These facts are typical features of $S_{3}$ flavor models accompanied by additional $U(1)$. In models without discrete flavor symmetry, $U(1)$ charge assignment generally has wider flexibility [20. On the other hand, models with only $S_{3}$ symmetry have to do with fermion mass hierarchy by arbitrarily tuning parameters such as Yukawa couplings and Higgs VEVs. In the following, we illustrate several examples of mass textures derived from $S_{3}$ and assistant $U(1)$ flavor dynamics, and also mention how to cure the above problems of charge assignment. It is noticed that mass hierarchy is dynamically realized in a similar way with a discrete subgroup of the flavor $U(1)$ symmetry. For example, all the hierarchical mass textures we discuss below can also be obtained by $Z_{N}$ subgroup with appropriate (enough large) $N$ and the same quantum numbers as in the $U(1)$ case. A smaller choice of $N$ would be possible and interesting from a viewpoint of brevity. In this case, the problem of fermion masses can be handled with flavor symmetries that are entirely discrete.

In what follows, we consider the cases that the flavor symmetries are broken at some high-energy scale, and renormalization-group running down to low energy should be taken into account if one obtains precise values of coupling constants, once the model below the symmetry-breaking scale is specified. However, it is not hard to see that, in all examples we discuss below, the running effects do not change qualitative results and can safely be dropped. The analysis in this section is performed in the models with a single flavor $S_{3}$ symmetry and the results are described in the complex basis, unless we particularly mention it.

\subsection{Nearest neighbor form}

The first example of $S_{3}$ models includes the mass texture proposed by Fritzsch [1]. We assign non-trivial $S_{3}$ charges to three-generation left- and right-handed fermions $\psi_{L i}$ and $\psi_{R j}(i=1,2,3)$. They are given by $\left(\psi_{L 1}, \psi_{L 2}\right)+\psi_{L 3}$ and $\left(\psi_{R 1}, \psi_{R 2}\right)+\psi_{R 3}$, namely, the first and second generations make up $S_{3}$ doublets and the third ones are singlets.

For the Higgs profile, we introduce an $S_{3}$-doublet $H_{D}=\left(H_{1}, H_{2}\right)$ as only a scalar with non-trivial $S_{3}$ charge. As mentioned in Section 3, $H_{D}$ has two possibilities concerned with the electroweak charge. We here take $H_{D}$ as a singlet of the electroweak gauge symmetry, and accordingly utilize the usual $S U(2)_{W}$-doublet Higgs $h$. Advantages of this 
choice are the suppression of flavor-changing rare processes, the preservation of gauge coupling unification in supersymmetric theory, and so on. A key point is that the VEV of the $S_{3}$ doublet scalar is assumed to take the form $\left\langle H_{D}\right\rangle=\left(\left\langle H_{1}\right\rangle, 0\right)$. This form can be dynamically justified by analyzing the scalar potential, as we will show in later section. Another type of VEV, $\left\langle H_{D}\right\rangle=\left(0,\left\langle H_{2}\right\rangle\right)$, just gives the case easily found by exchanging the up and down components in $S_{3}$ doublets.

The next step is to impose extra symmetries. As a simple example, we here assume a $Z_{2}$ parity acting non-trivially on $S_{3}$ doublets ("doublet parity"). We also have flavor $U(1)$ symmetry in order to control mass hierarchy and mixing among the three generations. Notice that due to the assignment of these non-vanishing charges the resultant mass matrices are simplified. That is, either a Higgs field or its complex conjugate can appear in each matrix element. At this stage, the $3 \times 3$ mass matrix for $\psi_{L, R}$ is found to be

$$
\left(\begin{array}{ccc} 
& a & \\
a & & b\left\langle H_{1}\right\rangle \\
& c\left\langle H_{1}\right\rangle & d
\end{array}\right) h,
$$

where $a, b, c$, and $d$ are the $\mathcal{O}(1)$ coefficients which include coupling constants and, if any, scalar fields with non-vanishing VEVs. Throughout this paper, $\mathcal{O}(1)$ coefficients are denoted where the fundamental mass scale of theory is taken to be unity. The texture form (4.1) was first adopted by Fritzsch [1] for quark mass matrices. It is noticed that the parity leads to some of vanishing matrix elements at tree level. An alternative parity assignment is viable where $\psi_{L 3}, \psi_{R 3}$ and $H_{D}$ have negative parity. A more interesting possibility is to incorporate supersymmetry in the model. In this case, vanishing matrix elements could be due to the holomorphicity of superpotential, combined with $U(1)$ quantum charges for realizing mass hierarchy, i.e. supersymmetric texture zeros.

To generate fermion mass hierarchy, we use the flavor $U(1)$ symmetry whose quantum charges are assigned as in the table below:

\begin{tabular}{c|ccccccc} 
& $\psi_{L 1,2}$ & $\psi_{L 3}$ & $\psi_{R 1,2}$ & $\psi_{R 3}$ & $h$ & $H_{D}$ & $\varphi$ \\
\hline$U(1)$ & $x$ & $x^{\prime}$ & $y$ & $y^{\prime}$ & 0 & $z$ & -1 \\
$Z_{2}$ & - & + & - & + & + & - & +
\end{tabular}

We have introduced a scalar field $\varphi$ to describe symmetry-invariant higher-dimensional operators which effectively induce mass terms below the $U(1)$ breaking scale $\langle\varphi\rangle$. The $U(1)$ charges are normalized by letting $\varphi$ take a unit charge, and $h$ is assumed to be neutral in order to fix overall mass scale. As a result, the orders of magnitude of the matrix elements in (4.1) becomes

$$
\left(\begin{array}{ccc} 
& \lambda^{x+y} & \\
\lambda^{x+y} & & \lambda^{x^{\prime}+y+z+\alpha} \\
& \lambda^{x+y^{\prime}+z+\alpha} & \lambda^{x^{\prime}+y^{\prime}}
\end{array}\right) h,
$$


where we have defined $\langle\varphi\rangle \equiv \lambda$ and $\left\langle H_{1}\right\rangle \equiv \lambda^{\alpha}$ ( $\alpha \geq 0$ for a consistent theory below the fundamental scale).

The eigenvalues of (4.2) are easily found; $m_{1} \sim h \lambda^{x+y-2(z+\alpha)}, m_{2} \sim h \lambda^{x+y+2(z+\alpha)}$, and $m_{3} \sim h \lambda^{x^{\prime}+y^{\prime}}$. To obtain a hierarchy $m_{1} \ll m_{2}$, the condition $z+\alpha<0$ is naively needed. It should be, however, noticed that this condition causes a problem that higherdimensional operators involving $H_{D}$ give larger effects and are not necessarily negligible. We will in the below enumerate possible resolutions to the problem; (i) to set $z+\alpha>0$, (ii) to take $z<0$ and make higher-dimensional operators negligible by supersymmetry, (iii) to tolerate some of higher-dimensional operators, (iv) to impose extra symmetry, (v) to change the $S_{3}$ representations of fermions, and (vi) to introduce $S_{3}$-singlet scalars.

(i) If one chooses $z+\alpha>0$, problematic non-renormalizable operators become irrelevant. This may be the simplest solution in the viewpoint of $U(1)$ charge assignment. It is found from the matrix form (4.2) that the mixing angle between the first and second generations becomes $\pi / 2$. Note here that the $S_{3}$ freedom cannot be used to reorder the mass eigenvalues because the $S_{3}$ group basis has already been fixed such that $H_{D}$ takes a particular form of VEV. While we know the generation mixing in the quark sector is small, the $S_{3}$ realization of the Fritzsch ansatz can be consistent (for $z+\alpha>0$ ), provided that the up and down quark sectors have almost the same flavor structure. Namely, If both the up and down sectors employ the matrix form (4.2), the label exchanging effects are cancelled out between the two sectors, and the quark mixing angles become small of powers of $\lambda$. Such a situation is similar to the case of the democratic quark mass matrices [14.

(ii) If one chooses $z<0$, higher-dimensional operators can be forbidden by holomorphicity in supersymmetric theory. For this being achieved, it is a simple assignment that all the $U(1)$ charges except for $H_{D}$ and $\varphi$ are positive in order not to give any higherdimensional terms. Since the doublet scalar $H_{D}$ now develops a VEV in the up component, the down component of doublet in the product $\left(H_{D}\right)^{2}$ becomes nonzero, and the singlet component in $\left(H_{D}\right)^{3}$ does [see the tensor product (2.7)]. Consequently, higher-order terms involving the products of $H_{D}$ might spoil the first-order analysis, because $H_{D}$ has a negative $U(1)$ charge $(z<0)$. In fact, the 1-3, 3-1, and 2-2 elements in the matrix (4.1) receive non-negligible contributions from $\left(H_{D}\right)^{2}$. The contributions to the 1-3 and 3-1 elements are forbidden by the doublet parity. We however find that higher-order contribution to the 2-2 element is difficult to be suppressed as long as the 2-3 and 3-2 matrix elements are allowed. A simple way to remedy this last problem is to add some extra symmetry, otherwise to apply the option (iii).

(iii) One, in some sense, negative choice is to abandon the exact Fritzsch ansatz and put up with some contribution from non-renormalizable operators. As mentioned above, 
a non-vanishing 2-2 element often appears even if the parity invariance is imposed. One can therefore choose as the third option that higher-order terms are generally forbidden by parity and/or supersymmetry while the 2-2 element is not. For example, in supersymmetric approach, the $U(1)$ charges are needed to satisfy the mild constraints $x+y+z<0$, $x+y^{\prime}+z \geq 0, x^{\prime}+y+z \geq 0$, and $z<0$. If this is the case, we obtain a mass matrix of the form (4.1) corrected by a nonzero 2-2 element from a higher-dimensional operator involving $\left(H_{D}\right)^{2}$. Such a type of mass texture has been recently discussed [21] to be suitable for solving fermion mass problems including the neutrino physics.

(iv) If one chooses to introduce more additional symmetries, harmful higher-dimensional operators might be removed. However such an operation generally reduces to complicate the models and involve uncontrollable factors, which make the models unfavorable.

(v) Contrary to the above options (i)-(iv), one can choose to extend the model to include more fields in other representations of flavor symmetry. Let us consider an additional Higgs $H_{A}$ of pseudo $S_{3}$ singlet. The representations of matter fields are accordingly changed to $\left(\psi_{L 2}, \psi_{L 3}\right)+\psi_{L 1}$ and $\left(\psi_{R 2}, \psi_{R 3}\right)+\psi_{R 1}$, i.e. the second and third generations are $S_{3}$ doublets. It is easily found by constructing $S_{3}$-invariant terms that one still has a mass matrix of the Fritzsch ansatz, provided that the non-vanishing VEVs are given by $\left\langle H_{D}\right\rangle=\left(0,\left\langle H_{2}\right\rangle\right)$ and $\left\langle H_{A}\right\rangle$. An important difference between this and the above models is whether the parity symmetry is needed or not to suppress undesired matrix elements. In the model here, the Fritzsch ansatz is obtained without imposing any parities. To make the 1-1 element negligible, it is sufficient to take appropriate $U(1)$ charge assignment, since the first-generation fermions now belong to different $S_{3}$ representations from the others, and have different $U(1)$ charges. Note that the example here may not be applied to left-right symmetric cases due to the anti-symmetric matrix elements generated by a pseudo singlet (i.e. $M_{23}=-M_{32}$ ). However asymmetrical forms of mass texture (zeros) could provide phenomenologically interesting possibility.

(vi) Another choice of additional scalar fields is a singlet Higgs $H_{S}$. A reason to introduce such singlet scalars is to suppress bare Yukawa couplings [e.g. $a$ and $d$ in the matrix (4.1)]. They are induced from other operators involving $S_{3}$ singlet scalars with non-vanishing $U(1)$ charges. Once the singlet scalars obtain VEVs, the resultant mass matrix takes the same form as (4.1), but flavor-invariant non-renormalizable operators are different due to the non-vanishing charges of singlet scalars. ${ }^{\dagger}$ Let $s$ be the $U(1)$ charge of $H_{S}$, which must be positive as will be seen below. There are three possible ways to include the $H_{S}$ scalar: (a) The 3-3 element comes from an operator involving $H_{S}$. That needs the charge conditions $x^{\prime}+y^{\prime}<0$ and $x^{\prime}+y^{\prime}+s \geq 0$. Moreover, for the mass

\footnotetext{
${ }^{\dagger}$ In case that $S U(2)_{W}$-doublet Higgses belong to non-trivial representations of $S_{3}$, such effects of $H_{S}$ (the suppression of bare Yukawa terms) can always be taken into account.
} 
hierarchy being realized, an additional condition $2(z+\alpha)<s+\alpha_{s}$ is required, where $\left\langle H_{S}\right\rangle \equiv \lambda^{\alpha_{s}}$. It is interesting that the operators which contribute to the 2-2 element is automatically suppressed, and one does not need to rely on any additional symmetries. Furthermore if one chooses $s+\alpha_{s}<\frac{5}{2}(z+\alpha)\left[\right.$ or $\left.s+\alpha_{s}<3(z+\alpha)\right]$, corrections to the 1-3 and 3-1 [or 1-1] elements become negligibly small. It can be checked that all the above charge conditions are easily satisfied. (b) In this case, the charge conditions $x+y<0$ and $x+y+s \geq 0$ are needed for $H_{S}$ to appear in the 1-2 and 2-1 elements. A proper mass hierarchy is realized when $2(z+\alpha)<s+\alpha_{s}$, and the 1-3 and 3-1 elements are negligibly small if $s+\alpha_{s}<3(z+\alpha)$. Unlike in the case (a), these charge conditions do not suppress higher-dimensional contributions to the 1-1 and 2-2 elements, so some symmetry should be imposed. (c) All the 1-2, 2-1, and 3-3 elements contain the $H_{S}$ field. That requires $x+y<0, x^{\prime}+y^{\prime}<0, x+y+s \geq 0$, and $x^{\prime}+y^{\prime}+s \geq 0$. Moreover one should take $z+\alpha<s+\alpha_{s}$ in order for fermion mass hierarchy to be preserved. With these charge conditions, all the operators concerned with the 2-2 element are automatically suppressed. It is, however, found that the 1-1 element generally receives sizable contribution unless extra symmetry is imposed.

In this way, the nearest-neighbor form of mass matrices, including the well-known Fritzsch ansatz, is realized in the framework of $S_{3}$ flavor symmetry. Moreover the mass hierarchy among the three generations is also achieved by incorporating an additional $U(1)$ symmetry. An important and non-trivial issue is whether $U(1)$ charges can be assigned so that they are compatible with the non-abelian flavor symmetry. We have shown typical examples of assignments which not only produce mass hierarchy but also suppress non-renormalizable operators which tend to disturb the mass hierarchy in the first-order estimation.

\subsection{Next-nearest neighbor form}

In most models with $S_{3}$ flavor symmetry and also in the previous subsection, the first two light generations are assumed to compose $S_{3}$ doublets. While such an assignment may be favorable to some phenomenological issues e.g. for suppressing flavor-changing processes, it is not necessarily the unique choice for $S_{3}$ charges. One may easily imagine different, but somewhat unfamiliar, $S_{3}$ flavor structures. In the following, we show that $S_{3}$ models with such twisted generations are also relevant to constructing realistic flavor theory of quarks and leptons. As a simple example, we here discuss the ansatz for mass texture proposed in [3], which type of texture suggests that the first and third generation fermions make up $S_{3}$ doublets.

The procedure is completely parallel to that in the previous subsection. The key 
ingredients are the representations of matter and Higgs fields, the profile of Higgs VEVs, and additional symmetries. Consider the matter representation $\left(\psi_{L 1}, \psi_{L 3}\right)+\psi_{L 2}$ and $\left(\psi_{R 1}, \psi_{R 3}\right)+\psi_{R 2}$, i.e. the first and third generations are $S_{3}$ doublets. We also introduce an $S_{3}$-doublet Higgs scalar $H_{D}=\left(H_{1}, H_{2}\right)$, whose down component $H_{2}$ is assumed to develop a non-vanishing VEV. The electroweak gauge invariance is implemented by an $S U(2)_{W}$-doublet scalar $h$, which is a singlet of $S_{3}$. The setup leads to the following form of mass matrix;

$$
\left(\begin{array}{ccc} 
& & a \\
& b & \\
a & & c\left\langle H_{2}\right\rangle
\end{array}\right) h
$$

where 1-2 and 2-1 elements have been suppressed by imposing a $Z_{2}$ parity concerning the second generation; $\psi_{L 2}, \psi_{R 2} \rightarrow-\psi_{L 2},-\psi_{R 2}$. Similar suppression can also be obtained by use of holomorphicity in supersymmetric theory. At this stage, the coefficients $a, b$, and $c$ are supposed to contain coupling constants and scalar VEVs, whose natural sizes are $\mathcal{O}(1)$. The texture (4.3) has the form of the Giudice ansatz [3] for the up quark mass matrix. The coefficients $a$ and $b$ should be smaller than $c\left\langle H_{2}\right\rangle$ to properly describe mass hierarchy of fermions. That is realized in the present work by introducing flavor $U(1)$ symmetry and a charge-compensating scalar $\varphi$. Defining $U(1)$ quantum charges as in the following table,

\begin{tabular}{c|ccccccc} 
& $\psi_{L 1,3}$ & $\psi_{L 2}$ & $\psi_{R 1,3}$ & $\psi_{R 2}$ & $h$ & $H_{D}$ & $\varphi$ \\
\hline$U(1)$ & $x$ & $x^{\prime}$ & $y$ & $y^{\prime}$ & 0 & $z$ & -1 \\
$Z_{2}$ & + & - & + & - & + & + & +
\end{tabular}

we have the orders of magnitude of the matrix elements

$$
\left(\begin{array}{ccc} 
& \lambda^{x+y} \\
\lambda^{x^{\prime}+y^{\prime}} & \\
\lambda^{x+y} & & \lambda^{x+y+z+\alpha}
\end{array}\right) h
$$

where $\langle\varphi\rangle \equiv \lambda$, and $\alpha$ parametrizes the $\mathrm{VEV}$ of $S_{3}$ doublet $\left\langle H_{2}\right\rangle \equiv \lambda^{\alpha}$. It is obvious that a charge condition $z+\alpha<0$ is necessary for mass hierarchy without inducing maximal generation mixing. Notice that this is similar to the case of the Fritzsch ansatz where higher-dimensional operators involving the $S_{3}$ doublet scalar lead to significant modification of matrix form. Therefore also in the present case, one can apply the resolutions discussed in the previous subsection to have natural hierarchy of mass eigenvalues and mixing angles.

\subsection{Asymmetric textures}

A more unfamiliar but interesting case is that flavor charges are asymmetrically assigned to left- and right-handed fermions, which generally lead to asymmetrical forms of mass 
textures. In grand unification schemes, the mass matrix of up-type quarks is often assumed to be symmetric (exactly speaking, hermitian), which comes from the fact that, even in the minimal $S U(5)$ model, one-generation up-type quarks with both chiralities belong to a single multiplet of unified gauge symmetry. However this is not generally the case for other Dirac mass matrices. In particular, the present experimental data suggest that the leptonic flavor mixing is quite un-parallel to the quark mixing. This asymmetrical observation can be compatible with quark-lepton unification, if fermion mass textures take asymmetrical forms in the generation space [10]. There are also some classes of nonhermitian ansatze for quark mass matrices [22], which are consistent with the experimental data and cannot be transformed to the symmetric solutions previously found in Ref. 44. It is therefore worthwhile to investigate the dynamical realization of asymmetric mass textures with discrete flavor symmetry. A systematic study of asymmetric mass matrices for the up and down quarks has recently been performed, particularly paying attention to the connection to leptonic flavor mixing [23].

Among various types of viable asymmetric textures, we here present an $S_{3}$ flavor model which predicts the mass matrix proposed in [24]. This texture is relevant to the neutrino Dirac mass matrix and is interesting in that large lepton mixing is realized without any tuning of couplings, if there is a suitable hierarchy among right-handed neutrino masses. The texture indicates neither that the first-two generations are in doublet representation nor that left- and right-handed fermions have parallel assignments of $S_{3}$ charges. That leads us to consider highly asymmetric flavor structure: $\left(\psi_{L 1}, \psi_{L 3}\right)+\psi_{L 2}$ and $\left(\psi_{R 2}, \psi_{R 3}\right)+\psi_{R 1}$. We also have $S U(2)_{W}$-singlet scalars, $H_{S}$ and $H_{D}=\left(H_{1}, H_{2}\right)$, which are $S_{3}$ singlet and doublet, respectively. The down component $H_{2}$ of the doublet $H_{D}$ is assumed to develop a non-vanishing $\mathrm{VEV}$. The electroweak gauge invariance is maintained by introducing an $S U(2)_{W}$-doublet standard model Higgs $h$, which is a singlet of $S_{3}$. Consequently we obtain the following form of mass texture:

$$
\left(\begin{array}{ccc}
a\left\langle H_{2}\right\rangle & d & \\
& b\left\langle H_{2}\right\rangle & e\left\langle H_{S}\right\rangle \\
e\left\langle H_{S}\right\rangle & & c\left\langle H_{2}\right\rangle
\end{array}\right) h .
$$

It is interesting to note that no additional symmetry is required to eliminate unwanted nonzero matrix elements, contrary to the previous two examples. The coefficients $a, \cdots, e$ contain coupling constants whose natural sizes are $\mathcal{O}(1)$. Splitting the sizes of the coefficients is easily obtained, for example, by introducing flavor $U(1)$ symmetry and a charge-compensating scalar $\varphi$. A typical $U(1)$ charge assignment is given by

\begin{tabular}{c|cccccccc} 
& $\psi_{L 1,3}$ & $\psi_{L 2}$ & $\psi_{R 2,3}$ & $\psi_{R 1}$ & $h$ & $H_{D}$ & $H_{S}$ & $\varphi$ \\
\hline$U(1)$ & $-x$ & 0 & $y$ & $z$ & 0 & $x$ & $2 x$ & -1
\end{tabular}


with $x>y \geq 0$ and $z \geq 0$, and we obtain the mass texture of the form

$$
\left(\begin{array}{ccc}
\lambda^{z} & \lambda^{z} & \\
& \lambda^{x+y} & \lambda^{x+y} \\
\left(\lambda^{x+y}\right) & & \lambda^{y}
\end{array}\right) h .
$$

The parameter $\lambda$ denotes the ratio of the $\operatorname{VEV}\langle\varphi\rangle$ to the fundamental scale. Compared to the matrix in 24], a non-vanishing 3-1 element is generated. However one can easily find that such a tiny entry does not contribute both to the mass eigenvalues and mixing angles, though it is obliged to be included to respect the flavor symmetry.

It was discussed [24] that the texture (4.6) (without the 3-1 element) could be derived by making use of more than two continuous flavor symmetries. By contrast, the present approach with discrete flavor symmetry seems somewhat simpler. The texture form is totally controlled by a single $S_{3}$ symmetry, and then, the mass hierarchy is determined by extra assumption (here the $U(1)$ flavor symmetry).

Finally let us comment on the corrections from non-renormalizable operators. It is found that the above charge assignment suppresses the contribution of higher-dimensional operators to the 1-3 and 3-2 matrix elements, and the texture zeros are not destabilized. On the other hand, the correction to the 2-1 element could be as large as the other components in the second rows, i.e. $\mathcal{O}\left(\lambda^{x+y}\right)$. This result itself is not a disaster but the above zero texture should be modified. That is similar to the cases in the previous subsections where higher-dimensional operators involving $H_{D}$ could give significant effects on the first-order approximation. Therefore one is able to apply similar resolutions to remedy the problem. For example, if the $S_{3}$ doublet scalar $H_{D}$ has a non-trivial charge of some other symmetry, any problematic deformation of mass matrix is completely avoidable.

\subsection{Missing a right-handed neutrino}

Referring to the $S_{3}$ algebra presented in Section 2, the maximal irreducible representation is doublet. The triplet representation is reducible, while it may be suitable to describe three-generation fermions. Two fermions with the same standard model charges are also well handled with $S_{3}$ symmetry. While we have already known the existence of three repetitions of the standard model fermions, there is an idea that only two right-handed neutrinos are effectively included [25]. In this framework, a missing right-handed neutrino might be thought to decouple at a superheavy scale and regarded as a flavor singlet. $\ddagger$ The remaining two right-handed neutrinos are hence treated as a doublet. In this subsec-

\footnotetext{
${ }^{\ddagger}$ Such a singlet should be $1_{\mathrm{S}}$ to preserve $S_{3}$ symmetry in low-energy regime. The existence of the third right-handed neutrino would be needed to reconcile the lepton sector to the quark one in high-energy unification schemes.
} 
tion, we present a model with three left-handed and two right-handed neutrinos in the framework of $S_{3}$ flavor symmetry.

We would like here to stress that another important issue of flavor symmetry is to simultaneously control different sectors of flavor. For example, physical quark mixing angles are determined with a conspiracy of the up and down mass matrices, and lowenergy Majorana neutrino masses are derived from Dirac and right-handed Majorana masses with the seesaw mechanism [26]. In the following, we illustrate simultaneous treatment of two different types of mass textures, i.e. the Dirac and Majorana masses of neutrinos. A more realistic flavor model, including quarks and charged leptons, will be discussed in the next section.

Among various models with two right-handed neutrinos, we here focus on the texture ansatz proposed in [27] and its realization with $S_{3}$ flavor symmetry in the neutrino sector. The model contains three left-handed leptons $\psi_{L 1,2,3}$ and two right-handed ones $\psi_{R 1,2}$. We assign the flavor charges to these fermions such that $\left(\psi_{L 1}, \psi_{L 2}\right)$ and $\left(\psi_{R 1}, \psi_{R 2}\right)$ are $S_{3}$ doublets and $\psi_{L 3}$ a singlet (either of the $S_{3}$ singlet representations is possible). As for the Higgs sector, at least two types of scalars are introduced for controlling two different sectors of mass textures, though not necessarily required. We take, as an example, two scalar fields which are $S_{3}$ doublets and the standard gauge singlets; $H_{D}=\left(H_{1}, H_{2}\right)$ and $H_{D}^{\prime}=\left(H_{1}^{\prime}, H_{2}^{\prime}\right)$, whose VEVs generate Dirac and Majorana masses, respectively. For compensating electroweak gauge invariance, a usual $S U(2)_{W^{-}}$doublet Higgs $h$ is also included. A more economical choice might be to have $S U(2)_{W}$-doublet and $S_{3}$-doublet scalar fields. In this case, however, there may exist some problems, as discussed before, that gauge coupling unification must be non-trivially realized and large rates of flavorchanging processes generally spoil the models.

The $S_{3}$ invariant terms induce the neutrino Dirac mass $M_{\nu}$ and the right-handed Majorana mass $M_{R}$, which read from the generic expressions (3.2) and (3.4),

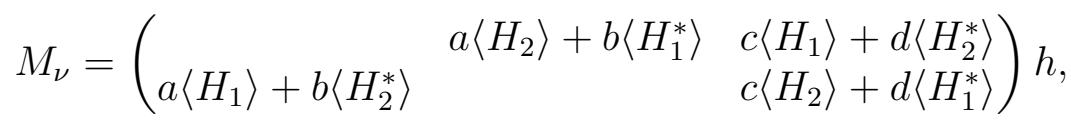

$$
\begin{aligned}
& M_{R}=\left(\begin{array}{ll}
e\left\langle H_{1}^{\prime}\right\rangle+f\left\langle H_{2}^{\prime *}\right\rangle & \\
& e\left\langle H_{2}^{\prime}\right\rangle+f\left\langle H_{1}^{\prime *}\right\rangle
\end{array}\right),
\end{aligned}
$$

where $a, \cdots, f$ are the coupling constants. Thus we have obtained the textures discussed in [27, if the generation indices are properly exchanged while physical consequences are unchanged (except for a sign reversion of as yet unobserved leptonic CP-violating quantity). The bi-large generation mixing in the neutrino sector is naturally established, provided that $\left(M_{\nu}\right)_{12} \simeq\left(M_{\nu}\right)_{13}$ and $\left(M_{\nu}\right)_{21} \simeq\left(M_{\nu}\right)_{23}$, and that there is a little hierarchy between these two combinations or between the mass eigenvalues of $M_{R}$ [27]. 
It is rather straightforward to incorporate supersymmetry into the above picture. Every field is promoted to a superfield which is in the same representations of the standard model gauge and flavor symmetries. The Dirac and Majorana mass terms are described by superpotential. Notice that the right-handed neutrinos $\psi_{R i}$ are embedded into the corresponding superfields in the form $\psi_{R i}^{c}$. As we explained in Section 3, this embedding modifies the tensor product and slightly changes the texture form. We thus find that the $S_{3}$-invariant superpotential induces the mass matrices

$$
M_{\nu}=\left(\begin{array}{lll}
a\left\langle H_{1}\right\rangle & & b\left\langle H_{2}\right\rangle \\
& a\left\langle H_{2}\right\rangle & b\left\langle H_{1}\right\rangle
\end{array}\right), \quad M_{R}=\left(\begin{array}{ll}
c\left\langle H_{1}^{\prime}\right\rangle & \\
& c\left\langle H_{2}^{\prime}\right\rangle
\end{array}\right) .
$$

Again the neutrino mass textures presented in [27] are dynamically realized in a supersymmetric $S_{3}$ model. It is easy to see that the coupling constants and scalar VEVs can be appropriately chosen for the neutrino physics without any fine tuning.

\section{Grand unification with flavor $S_{3}$}

Based on the above prescription for mass texture, in this section, we present a grand unified model of quarks and leptons with $S_{3}$ flavor symmetry. It is the most important point of flavor symmetry that any matrix form from flavor symmetry is valid only in the case that there are more than two sectors governed by the symmetry. A well-known example is the up and down quark mass textures whose forms are simultaneously altered by flavor rotation of $S U(2)_{W}$-doublet quarks. In other words, any realization of zero textures for a single sector cannot be physically distinguished from other freely-rotated, generally complicated, matrix forms. In the previous subsection, we discussed a model in which two types of neutrino mass matrices are controlled in the same fashion. To include the charged lepton sector is straightforward, referring to the general discussion of $S_{3}$-invariant matrix forms. Towards realistic flavor models of quarks and leptons, we here construct an illustrative grand unified model with $S_{3}$ flavor symmetry. The model has asymmetrical forms of mass textures due to non-trivial assignment of flavor charges, which particularly leads to bi-large lepton mixing. While the model presented here is not so complete, it suggests the validity of discrete symmetry for flavor physics and would give a large step to understand the origin of flavor.

Let us consider $S U(5)$ as a minimal candidate for unified gauge group. For matter Yukawa couplings, we introduce as usual the three-generation fermions, $\psi_{i}(10), \chi_{i}\left(5^{*}\right)$, $\nu_{i}(1)(i=1,2,3)$ and two scalars, $h_{u}(5)$ and $h_{d}\left(5^{*}\right)$. Needless to say, one needs some different types of scalar fields to break the unified gauge symmetry, but they are generally irrelevant to the Yukawa sector. We define the flavor $S_{3}$ charges of matter and scalar fields 
so that the combinations $\left(\psi_{1}, \psi_{2}\right),\left(\chi_{1}, \chi_{3}\right)$ and $\left(\nu_{1}, \nu_{2}\right)$ are $S_{3}$ doublets and the others in the $1_{\mathrm{S}}$ representation. To implement the $S_{3}$ invariance, a doublet and a singlet scalars are also introduced, called $H_{D}$ and $H_{S}$, respectively. The total field content is listed in the table below. We assume that $S_{3}$ is broken by the scalar VEVs; $\left\langle H_{D}\right\rangle=\left(\left\langle H_{1}\right\rangle, 0\right)$ and $\left\langle H_{S}\right\rangle$, which are of $\mathcal{O}(1)$ and can be the result of analyzing symmetry-invariant scalar potential without fine tuning.

\begin{tabular}{c|ccccccccccc} 
& $\psi_{1,2}$ & $\psi_{3}$ & $\chi_{1,3}$ & $\chi_{2}$ & $\nu_{1,2}$ & $\nu_{3}$ & $h_{u}$ & $h_{d}$ & $H_{D}$ & $H_{S}$ & $\varphi$ \\
\hline$S U(5)$ & 10 & 10 & $5^{*}$ & $5^{*}$ & 1 & 1 & 5 & $5^{*}$ & 1 & 1 & 1 \\
$S_{3}$ & 2 & 1 & 2 & 1 & 2 & 1 & 1 & 1 & 2 & 1 & 1 \\
$U(1)$ & 4 & 0 & 2 & 0 & $x+2$ & $y$ & -2 & -2 & 0 & 2 & -1
\end{tabular}

While the right-handed neutrinos are assigned to the $S_{3}$ representations $\left(\nu_{1}, \nu_{2}\right)+\nu_{3}$, that is not essential as long as the low-energy left-handed Majorana masses are concerned. It is because, in the seesaw mechanism, arbitrary rotations of right-handed neutrinos can be inserted while preserving left-handed Majorana mass matrix. In fact, the $S_{3}$ flavor charges (i.e. the flavor basis) of $\nu_{i}$ turn out to be fixed, once some other dynamics than $S_{3}$ is incorporated.

In addition to these, we have a $U(1)$ (or $Z_{N}$ ) symmetry to produce natural hierarchies among matrix elements (and also to help to discriminate $\nu_{i}$ ). A key of the mechanism is the existence of a scalar field $\varphi$ with a non-vanishing VEV, which is a bit smaller than the fundamental scale of the theory. The whole $S_{3}$ representations and $U(1)$ quantum numbers are shown in the table ( $x \geq 0$ and $2>y \geq 0$ for phenomenological requirements).

Let us first discuss the Majorana masses of right-handed neutrinos. They are given by the following form of $S_{3}$-invariant mass operator $\overline{\nu_{i}^{c}}\left(M_{R}\right)_{i j} \nu_{j}$ :

$$
M_{R}=\left(\begin{array}{ccc} 
& M & \\
M & & \\
& & M^{\prime}
\end{array}\right),
$$

where the mass parameters $M$ and $M^{\prime}$ are generally free. They contain some compound factors such as suppressions by flavor $U(1)$ invariance, but such suppression factors are cancelled out via the seesaw mechanism and irrelevant for low-energy quantity. The orders of magnitudes of $M$ and $M^{\prime}$ would be determined by experimental data. Theoretically this seems natural since $M_{R}$ cannot be directly induced by $h_{u, d}$, nor come from $H_{D, S}$, as the Majorana mass term violates lepton number symmetry. Otherwise one easily obtains $M_{R}$ by assuming VEVs of $S_{3}$-singlet scalar fields with lepton numbers. In any case, the most important point here is that a single $S_{3}$ flavor symmetry controls both Dirac and Majorana mass textures simultaneously. 
We also find that the $S_{3}$ and $U(1)$ flavor invariance leads to the following Dirac mass textures $M_{u, d, e, \nu}$ for quarks and leptons:

$$
\begin{gathered}
M_{u}=\left(\begin{array}{ccc}
\lambda^{6} & \lambda^{6} & \\
\lambda^{6} & & \lambda^{2} \\
& \lambda^{2} & 1
\end{array}\right) h_{u}, \quad M_{d}=\left(\begin{array}{ccc}
\lambda^{4} & & \lambda^{4} \\
\lambda^{4} & \lambda^{2} & \\
& & 1
\end{array}\right) h_{d}, \\
M_{e}=M_{d}^{\mathrm{T}}, \quad M_{\nu}=\left(\begin{array}{lll}
\lambda^{x+2} & & \lambda^{x+2} \\
\lambda^{x+2} & \lambda^{x} & \\
& \lambda^{y} & \lambda^{y}
\end{array}\right) h_{u},
\end{gathered}
$$

where we have neglected coupling constants and defined the expansion parameter $\lambda$ as the ratio of $\langle\varphi\rangle$ to the fundamental scale. Our first prediction obtained from the above textures is that the VEV ratio of two $S U(2)_{W}$ doublets should be large, that is, $h_{u} / h_{d} \sim$ $m_{t} / m_{b} \sim 60$. Secondly, we find the Majorana mass matrix $M_{L}$ of left-handed neutrinos derived from the seesaw formula

$$
M_{L}=\left(\begin{array}{ccc}
\epsilon & \\
\epsilon & 1 & 1+\epsilon \\
1+\epsilon & 1
\end{array}\right) \frac{\lambda^{2 y} h_{u}^{2}}{M^{\prime}} .
$$

Here the parameter $\epsilon$ has been defined as $\epsilon \equiv \lambda^{2 x-2 y+2}\left(M^{\prime} / M\right)$. The induced matrix elements in the 1-1, 1-3 and 3-1 positions are negligibly small and have been dropped in the expression (5.4). It is interesting to note that all the symbols " 1 " in the matrix (5.4) are exactly 1 due to the seesaw mechanism, and consequently, the lower-right $2 \times$ 2 sub-matrix has a reduced determinant of $\mathcal{O}(\epsilon)$, not $\mathcal{O}(1)$. For a small $\epsilon\left(\sim \lambda^{1-2}\right)$, the matrix (5.4) automatically realizes the bi-large generation mixing with hierarchical neutrino spectrum. The model also predicts a small lepton mixing of $\mathcal{O}(\epsilon)$ between the first and third generations. The planned improvements in the sensitivity to such a small angle are expected to reach $\mathcal{O}\left(10^{-2}\right)$ [28], and the above model will be testable in near future. On the other hand, the neutrinoless double beta decay [29] cannot be observed due to the negligible value of $\left(M_{L}\right)_{11}$.

It is found in the above Dirac mass matrices that some distorted values are induced for light generations, while the coefficients of matrix elements could be appropriately chosen for reproducing the observed values of fermion masses and mixing angles. We checked that, for the present simple field content, the flavor charge assignment of $S_{3}$ and $U(1)$ symmetries is uniquely determined for realizing the two conditions that (i) the right-handed neutrino masses $M_{R}$ come from the mass operator (5.1) and (ii) the rank of $2 \times 2$ sub-matrix of $M_{L}$ is reduced via the seesaw mechanism without tuning of couplings. Therefore an improvement of the above toy model is made by extending it to include additional scalar fields and modifying the texture forms. For example, the equivalence of 
the down and charged-lepton mass spectrum may be split by making use of Higgs fields in higher-dimensional representations. Another simple extension is to change the $S_{3}$ group basis, e.g. to the real basis. This might be a possible amelioration because the texture analysis and its physical consequences depend on the group basis of flavor symmetry, as explained before. We leave these issues to future investigation.

\section{Analysis of scalar potentials}

We have so far discussed mass textures of fermions in the framework of $S_{3}$ flavor symmetry, where the $S_{3}$ invariance is violated only by scalar VEVs. In particular, the assumption that either of components in a doublet has a vanishing VEV leads to texture forms for quark and lepton mass matrices, which result in various types of phenomenologically viable flavor models. In this section, we present, as an existence proof, the analysis of scalar potentials which generate desired forms of symmetry-breaking VEVs. We also discuss model parameters and symmetry for obtaining such VEVs. It should be noted that, unlike in the case of continuous flavor symmetry, a VEV of doublet cannot be transformed to an arbitrary form by $S_{3}$ rotations because of the discreteness of group operations (see Table 11). Even if there is a case that such rotation is useful, it is physically meaningful

only when other symmetries in the theory do not commute with $S_{3}$. This is not necessarily satisfied, for example, in the presence of $U(1)$ flavor symmetry.

\subsection{The general case}

We consider a single $S_{3}$ doublet scalar $H=\left(H_{1}, H_{2}\right)$ and analyze the structure of its generic scalar potential. The potential analysis becomes more involved if there are some numbers of scalar fields. However since we have shown that mass textures of quarks and leptons can be derived from a single doublet scalar, the analysis for one doublet would provide a step towards more practical cases with more than one doublets. Furthermore, when several scalar fields have VEVs of enough separated scales, they can be independently analyzed if the most generic terms are taken into account in scalar potentials.

The most general and renormalizable scalar potential for a doublet $H=\left(H_{1}, H_{2}\right)$ is given by

$$
\begin{aligned}
V\left(H_{1}, H_{2}\right)= & \mu^{2}\left(H_{1}^{\dagger} H_{1}+H_{2}^{\dagger} H_{2}\right)+\lambda\left[\left(H_{1}^{\dagger} H_{1}\right)^{2}+\left(H_{2}^{\dagger} H_{2}\right)^{2}\right] \\
& +\left[\rho^{2} H_{1} H_{2}+\alpha\left(H_{1}^{3}+H_{2}^{3}\right)+\beta\left(H_{1}^{2} H_{2}^{\dagger}+H_{2}^{2} H_{1}^{\dagger}\right)+\text { h.c. }\right] \\
& +\kappa H_{1}^{\dagger} H_{1} H_{2}^{\dagger} H_{2}+\left[\zeta H_{1}^{2} H_{2}^{2}+\eta\left(H_{1}^{\dagger} H_{1}^{2} H_{2}+H_{2}^{\dagger} H_{2}^{2} H_{1}\right)+\text { h.c. }\right]
\end{aligned}
$$


with respect to the $S_{3}$ invariance in the complex basis. The minimization of the potential leads to the following two conditions:

$$
\begin{gathered}
0=\frac{\partial V}{\partial H_{1}^{\dagger}=\mu^{2} H_{1}}+2 \lambda H_{1}^{\dagger} H_{1}^{2}+\rho^{2 *} H_{2}^{\dagger}+3 \alpha^{*} H_{1}^{\dagger 2}+\beta H_{2}^{2}+2 \beta^{*} H_{1}^{\dagger} H_{2}+\kappa H_{1} H_{2}^{\dagger} H_{2} \\
+2 \zeta^{*} H_{1}^{\dagger} H_{2}^{\dagger 2}+\eta H_{1}^{2} H_{2}+\eta^{*}\left(2 H_{1}^{\dagger} H_{1} H_{2}^{\dagger}+H_{2}^{\dagger 2} H_{2}\right) \\
0=\frac{\partial V}{\partial H_{2}^{\dagger}=\mu^{2} H_{2}}+2 \lambda H_{2}^{\dagger} H_{2}^{2}+\rho^{2 *} H_{1}^{\dagger}+3 \alpha^{*} H_{2}^{\dagger 2}+\beta H_{1}^{2}+2 \beta^{*} H_{1} H_{2}^{\dagger}+\kappa H_{1}^{\dagger} H_{1} H_{2} \\
+2 \zeta^{*} H_{1}^{\dagger 2} H_{2}^{\dagger}+\eta H_{1} H_{2}^{2}+\eta^{*}\left(H_{1}^{\dagger 2} H_{1}+2 H_{1}^{\dagger} H_{2}^{\dagger} H_{2}\right) .
\end{gathered}
$$

The minimum is ensured by examining whether the $2 \times 2$ matrix $\partial^{2} V / \partial H_{i} \partial H_{j}^{\dagger}(i, j=1,2)$ has positive eigenvalues at the extreme. Generally, the stationary conditions (6.2) and (6.3) are satisfied at various points in the field space. The origin $H_{1}=H_{2}=0$ is a solution of these two condition, but at this point, the $S_{3}$ flavor symmetry is unbroken and the result is unrealistic. Parallel to the well-known electroweak symmetry breaking, one easily avoids this trivial solution by assuming a negative value of $\mu^{2}$, which in turn makes the origin being a locally maximum point and destabilized. ${ }^{\S}$ A possible minimum we are interested in is that either of VEVs in a doublet scalar is vanishing, namely $H_{1} \neq 0$ and $H_{2}=0$, or vice versa. For $H_{2}=0$, the stationary conditions (6.2) and (6.3) become

$$
\begin{aligned}
\mu^{2} H_{1}+3 \alpha^{*} H_{1}^{\dagger 2}+2 \lambda H_{1}^{\dagger} H_{1}^{2} & =0 \\
\rho^{2} H_{1}+\beta^{*} H_{1}^{\dagger 2}+\eta H_{1}^{\dagger} H_{1}^{2} & =0 .
\end{aligned}
$$

It is found that the existence of non-trivial (at least local) minimum with $H_{1} \neq 0$ requires a set of conditions for the coupling constants. For example, $\rho=\beta=\eta=0$ or $\mu^{2} / \rho^{2}=$ $3 \alpha / \beta=2 \lambda / \eta$, and so on. The former condition could easily be obtained if the scalar has some other quantum charges than $S_{3}$. There exist various possibilities of coupling constants for stabilizing the scalar potential at non-trivial minimum. It is an intriguing task to find the conditions of couplings which are naturally realized with symmetries. Finally we comment on the VEV form $H_{1} \neq 0, H_{2} \neq 0$. Such a form of doublet VEVs is needed to achieve the texture form discussed in Section 4.4. If the coupling constants in the above scalar potential have the same order of magnitude (in the unit of fundamental scale), two VEVs $H_{1}$ and $H_{2}$ are on a similar order and generally have different values. In this case, it is difficult to write down the generic solution to (6.2) and (6.3) for the potential minimum. We numerically investigated that there indeed exists the minimum of the potential (6.1). As an example, a set of natural values of coupling constants, $\mu=\rho=\alpha=\beta \equiv m$ and $\lambda=\kappa=\zeta=\eta=1$, leads to the stationary point $H_{1}=0.61 \mathrm{~m}$

\footnotetext{
${ }^{\S}$ Exactly speaking, if $\mu^{2}<\left|\rho^{2}\right|$, the origin of field space is destabilized, but only along with limited directions.
} 
and $H_{2}=-1.62 \mathrm{~m}$. It can easily be checked that this point corresponds to a potential minimum by analyzing the second order derivatives of the potential. It is also interesting to explore the region of coupling constants for which such a form of doublet VEVs appears.

\subsection{Supersymmetric case}

The analysis is similarly performed in supersymmetric theory. For an $S_{3}$-doublet superfield $H=\left(H_{1}, H_{2}\right)$, the symmetry-invariant superpotential $W$ is given by

$$
W=m H_{1} H_{2}+\frac{y}{3}\left(H_{1}^{3}+H_{2}^{3}\right)+\frac{w}{2} H_{1}^{2} H_{2}^{2}+x H_{1} H_{2}\left(H_{1}^{3}+H_{2}^{3}\right)+\frac{z_{1}}{6}\left(H_{1}^{6}+H_{2}^{6}\right)+\frac{z_{2}}{3} H_{1}^{3} H_{2}^{3},
$$

where $m, y, \cdots, z_{1}, z_{2}$ are the coupling constants and we have included the most general operators with mass dimensions up to seven. In this subsection, we assume that supersymmetry is unbroken, that is, supersymmetry-breaking soft terms do not fix the breaking scale of flavor symmetry. The scalar potential is then given by

$$
\begin{aligned}
V\left(H_{1}, H_{2}\right)=\mid & m H_{1}+y H_{2}^{2}+w H_{1}^{2} H_{2}+x\left(4 H_{1} H_{2}^{3}+H_{1}^{4}\right)+z_{1} H_{2}^{5}+\left.z_{2} H_{1}^{3} H_{2}^{2}\right|^{2} \\
& +\left|m H_{2}+y H_{1}^{2}+w H_{1} H_{2}^{2}+x\left(4 H_{1}^{3} H_{2}+H_{2}^{4}\right)+z_{1} H_{1}^{5}+z_{2} H_{1}^{2} H_{2}^{3}\right|^{2}
\end{aligned}
$$

Comparing to the general case (6.1), we have the relations from supersymmetry:

$$
\mu^{2}=|m|^{2}, \quad \lambda=|y|^{2}, \quad \beta=m^{*} y, \quad \eta=m^{*} w, \quad \rho^{2}=\alpha=\kappa=\zeta=0 .
$$

We here focus on some simple cases obtained by introducing discrete symmetry on the superpotential. The vacuum is where one of the VEVs of doublet is vanishing, e.g. $H=\left(H_{1}, 0\right)$. Let us consider a $Z_{3}$ symmetry of which the superfield $H$ has a charge +1 . In this case, it permits only the $y, z_{1}$ and $z_{2}$ terms in the superpotential (6.6). As in the general case without supersymmetry, the origin $H_{1}=H_{2}=0$ is a trivial solution without flavor symmetry breaking. We here assume for simplicity that the origin is destabilized if supersymmetry-breaking (and $S_{3}$-breaking) soft scalar terms are included. We then find a potential minimum where the scalars develop their VEVs of the form $H=\left(\left(-y / z_{1}\right)^{1 / 3}, 0\right)$. At this vacuum, a vanishing VEV can give rise to mass textures (zeros) for quarks and leptons as explained before. Another simple example is a discrete $R$ symmetry $Z_{3 R}$ under which the superfield $H$ has a charge +1 . In this case, only the $m$ and $x$ terms are allowed in the superpotential (6.6). Similarly assuming that the origin does not correspond to the minimum, we find a vacuum at which the doublet has the one-sided form of VEVs $H=\left((-m / x)^{1 / 3}, 0\right)$. It may be interesting to note that the $Z_{3(R)}$ symmetry is suitable for discussing trilinear Yukawa terms and also for suppressing non-renormalizable operators involving more than one $S_{3}$ doublets. We finally comment that the analysis becomes more involved if higher-dimensional operators in Kähler terms were included. 


\section{Summary and discussions}

Discrete flavor symmetry is a powerful instrument in classifying and constructing mass matrix forms of quarks and leptons. It is greatly anticipated that such discrete symmetry is practically inherited from continuous symmetries in more fundamental theory in highenergy regime. In this paper, we focus on the $S_{3}$ group as a promising candidate of such symmetry. The $S_{3}$ is the smallest non-abelian discrete symmetry and also has a simple geometrical interpretation. After discussing some fundamental issues of the $S_{3}$ group (representations, tensor products and group basis dependence), we have presented the general forms of $3 \times 3$ mass matrices which are derived from various types of $S_{3}$ theories. Based on that prescription, we have performed the construction of particular ansatze of mass textures which have been often discussed in the literature. That includes asymmetrical matrices in generation space, which would be appropriate to discussing the leptonic flavor mixing.

Furthermore, discrete flavor symmetry can control the structure of mass matrices (texture) in dynamical ways. In particular, we have shown that vanishing matrix elements (texture zeros) are dynamically realized in the vacuum of scalar potential. It is however noted that texture zeros themselves do not give any explanation for fermion mass hierarchies. The observed values of masses and mixing angles are obtained in our scheme by introducing a $U(1)$ or $Z_{N}$ symmetry. A non-trivial issue here is whether abelian charges can be assigned so that they are compatible with non-abelian flavor symmetry. We have described typical examples of charge assignments which not only produce mass hierarchy among the generations but also suppress higher-dimensional operators which tend to disturb mass hierarchies in the first-order estimation. Unlike previous approaches with the $S_{3}$ group, we do not assume hierarchical coupling constants nor sequential breaking of flavor symmetry. In realistic flavor models for quarks and leptons, it is important that several different sectors are simultaneously controlled by flavor symmetry. As an illustrative example, a grand unified model has been constructed with a single $S_{3}$ symmetry. Finally, we have also analyzed the scalar potentials for $S_{3}$-doublet scalars, whose form of VEV is a key ingredient of our approach.

In this paper (especially below Section 4), we have focused on the models with a single $S_{3}$ symmetry. It may be an interesting task to explore other types of flavor extensions, e.g. $S_{3 L} \times S_{3 R}$ and other discrete symmetries larger than $S_{3}$. They would generally lead to different types of mass textures and physical consequences such as flavor-violating processes and their characteristic experimental signatures. More detailed study, including these issues, is left to future investigations. 


\section{Acknowledgments}

We would like to thank M. Tanimoto for helpful discussion. This work is supported by scientific grants from the Ministry of Education (No. 16028214, 16540258, 17740150) and by grant-in-aid for the scientific research on priority area "Progress in elementary particle physics of the 21st century through discoveries of Higgs boson and supersymmetry" (No. 16081209). 


\section{References}

[1] H. Fritzsch, Phys. Lett. B73 (1978) 317.

[2] H. Georgi and C. Jarlskog, Phys. Lett. B86 (1979) 297.

[3] G.F. Giudice, Mod. Phys. Lett. A7 (1992) 2429.

[4] P. Ramond, R.G. Roberts and G.G. Ross, Nucl. Phys. B406 (1993) 19.

[5] P.H. Frampton, S.L. Glashow and D. Marfatia, Phys. Lett. B536 (2002) 79.

[6] Y. Fukuda et al., Phys. Lett. B433 (1998) 9; ibid. B436 (1998) 33; Phys. Rev. Lett. 81 (1998) 1562; ibid. 82 (1999) 2644; ibid. 85 (2000) 3999.

[7] S. Fukuda et al., Phys. Rev. Lett. 86 (2001) 5651; ibid. 86 (2001) 5656; Phys. Lett. B539 (2002) 179; Q.R. Ahmad et al., Phys. Rev. Lett. 89 (2002) 011301; ibid. 89 (2002) 011302; K. Eguchi et al., Phys. Rev. Lett. 90 (2003) 021802; T. Araki et al., Phys. Rev. Lett. 94 (2005) 081801.

[8] S.F. King, Phys. Lett. B439 (1998) 350; JHEP 0209 (2002) 011; S. Davidson and S.F. King, Phys. Lett. B445 (1998) 191.

[9] A. Santamaria and J.W.F. Valle, Phys. Lett. B195 (1987) 423; R. Hempfling, Nucl. Phys. B478 (1996) 3; F.M. Borzumati, Y. Grossman, E. Nardi and Y. Nir, Phys. Lett. B384 (1996) 123; M. Drees, S. Pakvasa, X. Tata and T. ter Veldhuis, Phys. Rev. D57 (1998) 5335; E.J. Chun, S.K. Kang, C.W. Kim and U.W. Lee, Nucl. Phys. B544 (1999) 89; V. Bednyakov, A. Faessler and S. Kovalenko, Phys. Lett. B442 (1998) 203; B. Mukhopadhyaya, S. Roy and F. Vissani, Phys. Lett. B443 (1998) 191; O.C.W. Kong, Mod. Phys. Lett. A14 (1999) 903; J. Ferrandis, Phys. Rev. D60 (1999) 095012; D.E. Kaplan and A.E. Nelson, JHEP 0001 (2000) 033; A.S. Joshipura and S.K. Vempati, Phys. Rev. D60 (1999) 111303; A. Abada and M. Losada, Nucl. Phys. B585 (2000) 45; M. Hirsch, M.A. Diaz, W. Porod, J.C. Romao and J.W.F. Valle, Phys. Rev. D62 (2000) 113008 [Erratum-ibid. D65 (2002) 119901]; S. Davidson and M. Losada, Phys. Rev. D65 (2002) 075025.

[10] K.S. Babu and S.M. Barr, Phys. Lett. B381 (1996) 202; J. Sato and T. Yanagida, Phys. Lett. B430 (1998) 127; C.H. Albright, K.S. Babu and S.M. Barr, Phys. Rev. Lett. 81 (1998) 1167; J.K. Elwood, N. Irges and P. Ramond, Phys. Rev. Lett. 81 (1998) 5064; Y. Nomura and T. Yanagida, Phys. Rev. D59 
(1999) 017303; N. Haba, Phys. Rev. D59 (1999) 035011; G. Altarelli and F. Feruglio, Phys. Lett. B451 (1999) 388; W. Buchmuller and T. Yanagida, Phys. Lett. B445 (1999) 399; Z. Berezhiani and A. Rossi, JHEP 9903 (1999) 002; M. Bando and T. Kugo, Prog. Theor. Phys. 101 (1999) 1313; S. Lola and G. G. Ross, Nucl. Phys. B553 (1999) 81; Y. Nir and Y. Shadmi, JHEP 9905 (1999) 023; K. Yoshioka, Mod. Phys. Lett. A15 (2000) 29; P.H. Frampton and A. Rasin, Phys. Lett. B478 (2000) 424; M. Bando, T. Kugo and K. Yoshioka, Prog. Theor. Phys. 104 (2000) 211; Phys. Lett. B483 (2000) 163; J.M. Mira, E. Nardi, D.A. Restrepo and J.W.F. Valle, Phys. Lett. B492 (2000) 81; M. Bando et al., Phys. Rev. D63 (2001) 113017; N. Haba and H. Murayama, Phys. Rev. D63 (2001) 053010; M.S. Berger and K. Siyeon, Phys. Rev. D63 (2001) 057302; A. Kageyama, M. Tanimoto and K. Yoshioka, Phys. Lett. B512 (2001) 349; K.S. Babu and S. M. Barr, Phys. Lett. B525 (2002) 289; X.J. Bi and Y.B. Dai, Eur. Phys. J. C27 (2003) 43; K.S. Babu, I. Gogoladze and K. Wang, Nucl. Phys. B660 (2003) 322; R. Kitano and T. j. Li, Phys. Rev. D67 (2003) 116004; T. Asaka, Phys. Lett. B562 (2003) 291; K.S. Babu, T. Enkhbat and I. Gogoladze, Nucl. Phys. B678 (2004) 233.

[11] J. Sato, K. Tobe and T. Yanagida, Phys. Lett. B498 (2001) 189; J. Sato and K. Tobe, Phys. Rev. D63 (2001) 116010.

[12] A.Y. Smirnov, Phys. Rev. D48 (1993) 3264; Nucl. Phys. B466 (1996) 25; M. Tanimoto, Phys. Lett. B345 (1995) 477; M. Bando, T. Kugo and K. Yoshioka, Phys. Rev. Lett. 80 (1998) 3004; M. Jezabek and Y. Sumino, Phys. Lett. B440 (1998) 327; M. Bando and K. Yoshioka, Prog. Theor. Phys. 100 (1998) 1239; D. Falcone, Phys. Rev. D61 (2000) 097302; E.K. Akhmedov, G.C. Branco and M.N. Rebelo, Phys. Lett. B478 (2000) 215; T.K. Kuo, G.H. Wu and S.W. Mansour, Phys. Rev. D61 (2000) 111301; A. Datta, F.S. Ling and P. Ramond, Nucl. Phys. B671 (2003) 383; M. Honda, S. Kaneko and M. Tanimoto, Phys. Lett. B593 (2004) 165; R. Dermisek, Phys. Rev. D70 (2004) 073016; M. Lindner, M.A. Schmidt and A.Y. Smirnov, JHEP 0507 (2005) 048.

[13] E. Derman and H.S. Tsao, Phys. Rev. D20 (1979) 1207; Y. Yamanaka, H. Sugawara and S. Pakvasa, Phys. Rev. D25 (1982) 1895 [Erratum-ibid. D29 (1984) 2135]; G. Ecker, Z. Phys. C24 (1984) 353; D. Chang, W. Y. Keung and G. Senjanovic, Phys. Rev. D42 (1990) 1599; D.B. Kaplan and M. Schmaltz, Phys. Rev. D49 (1994) 3741; P.H. Frampton and T.W. Kephart, Int. J. Mod. Phys. A10 (1995) 4689; M. Schmaltz, Phys. Rev. D52 (1995) 1643; P.H. Frampton and O.C.W. Kong, Phys. Rev. Lett. 77 (1996) 1699; A. Aranda, C.D. Carone and R.F. Lebed, 
Phys. Lett. B474 (2000) 170; E. Ma and G. Rajasekaran, Phys. Rev. D64 (2001) 113012; K.S. Babu, E. Ma and J.W.F. Valle, Phys. Lett. B552 (2003) 207; E. Ma, Mod. Phys. Lett. A17 (2002) 2361; W. Grimus and L. Lavoura, Phys. Lett. B572 (2003) 189; C.I. Low and R.R. Volkas, Phys. Rev. D68 (2003) 033007; M. Hirsch, J.C. Romao, S. Skadhauge, J.W.F. Valle and A. Villanova del Moral, Phys. Rev. D69 (2004) 093006; W. Grimus, A.S. Joshipura, S. Kaneko, L. Lavoura and M. Tanimoto, JHEP 0407 (2004) 078; M. Frigerio, S. Kaneko, E. Ma and M. Tanimoto, Phys. Rev. D71 (2005) 011901; K.S. Babu and J. Kubo, Phys. Rev. D71 (2005) 056006; G. Altarelli and F. Feruglio, Nucl. Phys. B720 (2005) 64; A. Zee, Phys. Lett. B630 (2005) 58.

[14] H. Harari, H. Haut and J. Weyers, Phys. Lett. B78 (1978) 459; Y. Koide, Phys. Rev. D28 (1983) 252; ibid. D39 (1989) 1391; P. Kaus and S. Meshkov, Mod. Phys. Lett. A3 (1988) 1251 [Erratum-ibid. A4 (1989) 603]; L. Lavoura, Phys. Lett. B228 (1989) 245; M. Tanimoto, Phys. Rev. D41 (1990) 1586; G.C. Branco, J.I. SilvaMarcos and M.N. Rebelo, Phys. Lett. B237 (1990) 446; H. Fritzsch and J. Plankl, Phys. Lett. B237 (1990) 451; H. Fritzsch and Z.Z. Xing, Phys. Lett. B372 (1996) 265; M. Fukugita, M. Tanimoto and T. Yanagida, Phys. Rev. D57 (1998) 4429; R.N. Mohapatra and S. Nussinov, Phys. Lett. B441 (1998) 299; P.F. Harrison and W.G. Scott, Phys. Lett. B557 (2003) 76; F. Caravaglios and S. Morisi, arXiv:hep-ph/0503234.

[15] S. Pakvasa and H. Sugawara, Phys. Lett. B73 (1978) 61; E. Derman, Phys. Rev. D19 (1979) 317; L.J. Hall and H. Murayama, Phys. Rev. Lett. 75 (1995) 3985; C.D. Carone, L.J. Hall and H. Murayama, Phys. Rev. D53 (1996) 6282; R.N. Mohapatra, A. Perez-Lorenzana and C.A. de Sousa Pires, Phys. Lett. B474 (2000) 355; J. Kubo, A. Mondragon, M. Mondragon and E. Rodriguez-Jauregui, Prog. Theor. Phys. 109 (2003) 795; T. Kobayashi, J. Kubo and H. Terao, Phys. Lett. B568 (2003) 83; J. Kubo, H. Okada and F. Sakamaki, Phys. Rev. D70 (2004) 036007; T. Araki, J. Kubo and E.A. Paschos, Eur. Phys. J. C45 (2006) 465.

[16] D. Wyler, Phys. Rev. D19 (1979) 330; E. Ma, Phys. Rev. D43 (1991) 2761; N.G. Deshpande, M. Gupta and P.B. Pal, Phys. Rev. D45 (1992) 953; R. Dermisek and S. Raby, Phys. Rev. D62 (2000) 015007; S.L. Chen, M. Frigerio and E. Ma, Phys. Rev. D70 (2004) 073008 [Erratum-ibid. D70 (2004) 079905]; W. Grimus and L. Lavoura, JHEP 0508 (2005) 013.

[17] S. Dimopoulos and H. Georgi, Nucl. Phys. B193 (1981) 150; J.R. Ellis and D.V. Nanopoulos, Phys. Lett. B110 (1982) 44; J.F. Donoghue, H.P. Nilles and 
D. Wyler, Phys. Lett. B128 (1983) 55; L.J. Hall, V.A. Kostelecky and S. Raby, Nucl. Phys. B267 (1986) 415; F. Gabbiani and A. Masiero, Nucl. Phys. B322 (1989) 235; J.S. Hagelin, S. Kelley and T. Tanaka, Nucl. Phys. B415 (1994) 293; F. Gabbiani, E. Gabrielli, A. Masiero and L. Silvestrini, Nucl. Phys. B477 (1996) 321.

[18] A.H. Chamseddine, R. Arnowitt and P. Nath, Phys. Rev. Lett. 49 (1982) 970; R. Barbieri, S. Ferrara and C.A. Savoy, Phys. Lett. B119 (1982) 343; L.J. Hall, J. Lykken and S. Weinberg, Phys. Rev. D27 (1983) 2359; N. Ohta, Prog. Theor. Phys. 70 (1983) 542; H.P. Nilles, Phys. Rept. 110 (1984) 1.

[19] C.D. Froggatt and H.B. Nielsen, Nucl. Phys. B147 (1979) 277.

[20] M. Leurer, Y. Nir and N. Seiberg, Nucl. Phys. B420 (1994) 468; L.E. Ibanez and G.G. Ross, Phys. Lett. B332 (1994) 100; E. Dudas, S. Pokorski and C.A. Savoy, Phys. Lett. B356 (1995) 45; P. Binetruy, S. Lavignac and P. Ramond, Nucl. Phys. B477 (1996) 353; R.N. Mohapatra and A. Riotto, Phys. Rev. D55 (1997) 4262; K. Choi, E.J. Chun and H.D. Kim, Phys. Lett. B394 (1997) 89; A.E. Nelson and D. Wright, Phys. Rev. D56 (1997) 1598; Y. Grossman, Y. Nir and Y. Shadmi, JHEP 9810 (1998) 007; S.F. King, Nucl. Phys. B576 (2000) 85; Q. Shafi and Z. Tavartkiladze, Phys. Lett. B482 (2000) 145; N. Maekawa, Prog. Theor. Phys. 106 (2001) 401; N. Maekawa and T. Yamashita, Prog. Theor. Phys. 107 (2002) 1201; M. Kakizaki and M. Yamaguchi, JHEP 0206 (2002) 032; H.K. Dreiner, H. Murayama and M. Thormeier, Nucl. Phys. B729 (2005) 278; P.H. Chankowski, K. Kowalska, S. Lavignac and S. Pokorski, Phys. Rev. D71 (2005) 055004.

[21] D.s. Du and Z.z. Xing, Phys. Rev. D48 (1993) 2349; H. Fritzsch and Z.z. Xing, Phys. Lett. B353 (1995) 114; P.S. Gill and M. Gupta, Phys. Rev. D56 (1997) 3143; J.L. Chkareuli and C.D. Froggatt, Phys. Lett. B450 (1999) 158; H. Nishiura, K. Matsuda and T. Fukuyama, Phys. Rev. D60 (1999) 013006; D. Falcone and F. Tramontano, Phys. Rev. D63 (2001) 073007; R. Rosenfeld and J.L. Rosner, Phys. Lett. B516 (2001) 408; W. Buchmuller and D. Wyler, Phys. Lett. B521 (2001) 291; J.L. Chkareuli, C.D. Froggatt and H.B. Nielsen, Nucl. Phys. B626 (2002) 307; M. Bando and M. Obara, Prog. Theor. Phys. 109 (2003) 995.

[22] G.C. Branco and J.I. Silva-Marcos, Phys. Lett. B331 (1994) 390; T.K. Kuo, S.W. Mansour and G.H. Wu, Phys. Rev. D60 (1999) 093004; G.C. Branco, D. Emmanuel-Costa and R. Gonzalez Felipe, Phys. Lett. B477 (2000) 147.

[23] N. Uekusa, A. Watanabe and K. Yoshioka, Phys. Rev. D71 (2005) 094024. 
[24] G. Altarelli, F. Feruglio and I. Masina, Phys. Lett. B472 (2000) 382.

[25] R. Kuchimanchi and R.N. Mohapatra, Phys. Rev. D66 (2002) 051301; T. Endoh, S. Kaneko, S.K. Kang, T. Morozumi and M. Tanimoto, Phys. Rev. Lett. 89 (2002) 231601; M. Raidal and A. Strumia, Phys. Lett. B553 (2003) 72; S.F. King, Phys. Rev. D67 (2003) 113010; S. Raby, Phys. Lett. B561 (2003) 119; B. Dutta and R.N. Mohapatra, Phys. Rev. D68 (2003) 056006; V. Barger, D.A. Dicus, H.J. He and T.j. Li, Phys. Lett. B583 (2004) 173; W.l. Guo and Z.z. Xing, Phys. Lett. B583 (2004) 163; W. Rodejohann, Eur. Phys. J. C32 (2004) 235; A. Ibarra and G.G. Ross, Phys. Lett. B591 (2004) 285; S.h. Chang, S.K. Kang and K. Siyeon, Phys. Lett. B597 (2004) 78.

[26] T. Yanagida, in Proceedings of the Workshop on Unified Theories and Baryon Number in the Universe, eds. O. Sawada and A. Sugamoto (KEK report 79-18, 1979); M. Gell-Mann, P. Ramond and R. Slansky, in Supergravity, eds. P. van Nieuwenhuizen and D.Z. Freedman (North Holland, Amsterdam, 1979).

[27] P.H. Frampton, S.L. Glashow and T. Yanagida, Phys. Lett. B548 (2002) 119.

[28] A. Cervera, A. Donini, M.B. Gavela, J.J. Gomez Cadenas, P. Hernandez, O. Mena and S. Rigolin, Nucl. Phys. B579 (2000) 17; P. Huber, M. Lindner and W. Winter, Nucl. Phys. B645 (2002) 3; P. Huber, M. Lindner, T. Schwetz and W. Winter, Nucl. Phys. B665 (2003) 487.

[29] For a recent review, see, S.R. Elliott and P. Vogel, Ann. Rev. Nucl. Part. Sci. 52 (2002) 115 [arXiv: hep-ph/0202264. 